Illinois State University

ISU ReD: Research and eData

Theses and Dissertations

3-27-2017

\title{
Comparison of Clinician Assisted versus Unassisted PNF Stretching on Hamstring Flexibility, Isometric Force Output, and Maximum Vertical Jump Height
}

Joel T. Lockhart

Illinois State University, jtlock1@ilstu.edu

Follow this and additional works at: https://ir.library.illinoisstate.edu/etd

Part of the Biomechanics Commons

\section{Recommended Citation}

Lockhart, Joel T., "Comparison of Clinician Assisted versus Unassisted PNF Stretching on Hamstring Flexibility, Isometric Force Output, and Maximum Vertical Jump Height" (2017). Theses and Dissertations. 723.

https://ir.library.illinoisstate.edu/etd/723

This Thesis is brought to you for free and open access by ISU ReD: Research and eData. It has been accepted for inclusion in Theses and Dissertations by an authorized administrator of ISU ReD: Research and eData. For more information, please contact ISUReD@ilstu.edu. 


\section{COMPARISON OF CLINICIAN ASSISTED VERSUS UNASSISTED PNF STRETCHING \\ ON HAMSTRING FLEXIBILITY, ISOMETRIC FORCE OUTPUT, AND \\ MAXIMUM VERTICAL JUMP HEIGHT}

Joel T. Lockhart

\section{Pages}

Introduction: Hamstring strains are among one of the most common acute injuries that occur in soccer players. Previous research has shown that reduced hamstring flexibility is a risk factor for injury, however this is modifiable through stretching. Some stretching techniques have contributed to decreased performance on measures important to success in sport. Proprioceptive neuromuscular facilitation (PNF) is one effective stretching technique to improve hamstring flexibility, but requires a trained partner or clinician to perform properly. It is unknown if the performance of PNF stretching without a clinician's assistance could be as effective. It is also unknown if PNF stretching affects soccer related performance outcomes of force output and vertical jump height. Objective: To determine the effects of clinician assisted versus unassisted contract-relax PNF stretching on hamstring flexibility, maximum voluntary isometric contraction (MVIC) of the hamstring muscle group, and athletic performance via vertical jump height.

Design: Controlled laboratory study. Participants: A sample of twenty-six physically active male and female college students with restricted hamstring flexibility, defined as limited passive hip flexion range of motion with the leg straight ( $<90$ degrees), participated in this study. Procedures: Subjects were randomly allocated to one of three groups; the clinician assisted, unassisted, or control groups following confirmation of inclusion criteria. The control group 
completed pre- and post- test measures only. The intervention groups completed a 6-week stretching intervention (12 total sessions) either with the assistance of a clinician or utilizing a stretching strap. Pre- and Post- intervention measurements were recorded for all outcome variables. Main Outcome Measures: Hip flexion range of motion was measured passively with the knee extended. Hamstring MVIC was assessed using a handheld dynamometer and normalized to each participant's body mass. Maximal vertical jump height was measured using a Vertec. Three trials were recorded for each measure and the arithmetic mean was used to calculate change scores (Post - Pre). Change scores were utilized for data analysis. Separate oneway ANOVA's were performed to identify differences between groups in the magnitude of change. Results: The one-way ANOVA for hip flexion ROM change scores was significant (F ${ }_{(2,}$ $\left.{ }_{25)}=9.853, \mathrm{p}=0.001\right)$, indicating there were significant differences in the amount of change in ROM when compared across groups. Post-hoc comparisons found both the Unassisted $\left(10.26^{\circ} \pm\right.$ $6.03, \mathrm{p}=0.002)$ and Assisted $\left(10.27^{\circ} \pm 3.81, \mathrm{p}=0.004\right)$ PNF intervention groups had a greater improvement in ROM compared to the Control group $\left(-0.41^{\circ} \pm 7.00\right)$. There were no differences between the amount of change in the Unassisted group compared to the Assisted group. The oneway ANOVA found no significant changes in $\operatorname{MVIC}\left(\mathrm{F}_{(2,25)}=2.542, \mathrm{p}=0.101\right)$, and vertical jump height $\left(\mathrm{F}_{(2,25)}=0.133, \mathrm{p}=0.877\right)$. Conclusion: Both the Assisted and Unassisted PNF stretching techniques were successful in improving hamstring flexibility. Performance measures were not impacted by the stretching intervention. Teaching a patient to perform the contractrelax PNF stretching technique to target hamstring flexibility is as effective as the clinician providing the assistance. These results may provide time saving benefits to clinicians while promoting improved flexibility and independence in patients.

KEYWORDS: PNF, Hamstring Flexibility, Soccer, Vertical Jump, Strength 
COMPARISON OF CLINICIAN ASSISTED VERSUS UNASSISTED PNF STRETCHING

ON HAMSTRING FLEXIBILITY, ISOMETRIC FORCE OUTPUT, AND

MAXIMUM VERTICAL JUMP HEIGHT

JOEL T. LOCKHART

A Thesis Submitted in Partial Fulfillment of the Requirements for the Degree of

MASTER OF SCIENCE

School of Kinesiology and Recreation

ILLINOIS STATE UNIVERSITY 
Copyright 2017 Joel T. Lockhart 
COMPARISON OF CLINICIAN ASSISTED VERSUS UNASSISTED PNF STRETCHING

ON HAMSTRING FLEXIBILITY, ISOMETRIC FORCE OUTPUT, AND

MAXIMUM VERTICAL JUMP HEIGHT

JOEL T. LOCKHART

COMMITTEE MEMBERS:

Rebecca L. Begalle, Chair

Skip M. Williams

Noelle M. Selkow 


\section{ACKNOWLEDGMENTS}

I would like to thank Dr. Begalle for her dedication, patience, and guidance in taking an idea and turning it into a fun Thesis project. Many thanks go to Dr. Williams and Dr. Selkow for their assistance and encouragement in the creation and completion of this Thesis project. Thank you to Bridget Hill and Matt Kovarik for your assistance in data collection and devoting your time and efforts to help complete this thesis project and best of luck with the next step. Much appreciation and many thanks go to the Graduate Athletic Training program at Illinois State University and the Athletic Training Staff at Illinois Wesleyan University for allowing me the opportunity to pursue a goal which turned into the experience of a lifetime. Thank you to my family, Karen, Jehan, Candy, Erin, Chad, Anthony, Eilish, Connor, Jeremy, Marissa, Ryan, Sam, Jene, and all those who entered my life through Athletic Training and/or are the friends that have become family. $\mathrm{T}$, this is for you so that you never have to worry. 


\section{CONTENTS}

Page

ACKNOWLEDGMENTS

$\begin{array}{ll}\text { CONTENTS } & \text { ii }\end{array}$

TABLES

FIGURES

CHAPTER

I. THE PROBLEM AND ITS BACKGROUND 1

Introduction 1

II. REVIEW OF LITERATURE 4

$\begin{array}{ll}\text { Anatomy } & 4\end{array}$

Hamstrings 4

Hamstring Strain: What Is It?

$\begin{array}{lr}\text { Stretching Mechanism } & 9\end{array}$

Proprioceptive Neuromuscular Facilitation: PNF 11

PNF Stretching Techniques 13

Effects on Maximum Voluntary Contraction $\quad 15$

Effects on Vertical Jump Height 15

Clinician-Assisted versus Self-Directed PNF Stretching 16

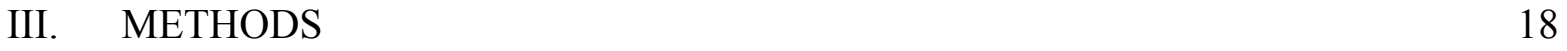

$\begin{array}{ll}\text { Research Design } & 18\end{array}$

$\begin{array}{ll}\text { Participants } & 18\end{array}$

$\begin{array}{ll}\text { Instrumentation } & 19\end{array}$

$\begin{array}{ll}\text { Procedures } & 20\end{array}$

Consenting Process \& Demographics 20

Screening Procedure: Passive Hip Flexion ROM 21

$\begin{array}{ll}\text { Pre-Test Measurements } & 22\end{array}$

Intervention 23

Post-Test Measurements 26 
$\begin{array}{ll}\text { Data Reduction } & 26\end{array}$

Statistical Analysis $\quad 26$

$\begin{array}{ll}\text { IV. RESULTS } & 30\end{array}$

$\begin{array}{ll}\text { V. DISCUSSION } & 31\end{array}$

$\begin{array}{ll}\text { REFERENCES } & 38\end{array}$

APPENDIX A: IRB INFORMED CONSENT 49

APPENDIX B: DATA COLLECTION SHEET

APPENDIX C: UNASSISTED PNF STRETCH FLYER 53 


\section{TABLES}

Table

Page

1. Magnitude of Change for Dependent Variables 


\section{FIGURES}

Figure $\quad$ Page

1. Placement of Digital Inclinometer 28

2. Placement of Handheld Dynamometer 28

3. Unassisted PNF Stretch Protocol 29

4. Assisted PNF Stretch Protocol 29 


\section{CHAPTER I}

\section{THE PROBLEM AND ITS BACKGROUND}

\section{Introduction}

Hamstring strains are among one of the most common acute injuries that occur in soccer players across all skill levels, accounting for $12-16 \%$ of all soccer injuries. ${ }^{1}$ A hamstring strain is described as posterior thigh pain without the involvement of direct contact to the leg. ${ }^{2}$ There are multiple factors that may contribute to hamstring strains such as previous injury, age, muscle strength imbalances, and poor flexibility. ${ }^{3-5}$ Specifically, previous research has shown that athletes with reduced hamstring flexibility are at an increased risk for a hamstring muscle injury. ${ }^{3}$ Flexibility is an intrinsic, modifiable risk factor that can be addressed through a variety of clinical interventions. ${ }^{1,5}$ The most widely used method of increasing hamstring flexibility is through stretching. ${ }^{6}$ Implementing a stretching program to increase the flexibility of the hamstring muscles would be one preventative measure to help reduce the risk of sustaining a hamstring strain. ${ }^{6,7}$

Proprioceptive neuromuscular facilitation (PNF) is one technique of stretching that has been effective in improving hamstring flexibility and contributing to musculotendinous injury prevention. ${ }^{5-11}$ PNF stretching is utilized to improve muscle elasticity and has been shown to have a positive effect on both active and passive ranges of motion in one day as well as six and seven week interventions. ${ }^{12-16}$

There are three PNF stretching techniques, 1) hold-relax, 2) contract-relax, and 3) slowreversal-hold-relax. ${ }^{6,12,17,18}$ Each utilize the contractions of different agonist and antagonist muscles and involve various contraction types, which are significant in the effectiveness of each technique. The current study will utilize the contract-relax PNF technique, which is a 
combination of passive hip flexion, followed by an isotonic hamstring contraction against resistance, and further passive hip flexion. ${ }^{19}$ Previous research has demonstrated that the contract-relax technique was effective in improving hamstring flexibility after an acute 5 day stretching intrevention. ${ }^{20}$

The effects of PNF stretching on hamstring flexibility, measured through hip flexion range of motion, are fairly well known. However, this form of stretching is time consuming and requires the hands on assistance of a clinician (or partner) that is educated in the proper performance of the technique. Previous research utilized a stretch strap, in place of a clinician, to have the patient provide a passive stretch and resistance during a PNF stretching protocol. ${ }^{10}$ Maddigan et al. ${ }^{10}$ compared static, partner-assisted and unassisted PNF stretching on hip range of motion, movement time, reaction time, and dynamic angular velocity. All forms of stretching were equally effective in improving range of motion measures, but significant decreases in the athletic measures of movement time and angular velocity were observed..$^{10}$ To our knowledge, the current study is the first to investigate the use of assisted versus unassisted PNF stretching over the course of a 6-week intervention on measures of flexibility and performance measures of force output and maximum vertical jump height. If athletes are able to perform a contract-relax PNF stretching technique independently using a stretch strap and have equal benefits to those who had access to a trained clinician, results of this study would be promising for impacting a larger number of athletes and potentially reducing injury rates. The focus of the current study is limited hamstring flexibility and its ability to be modified through rehabilitation interventions. We aim to study the effects of two variations of a PNF stretching technique on improving known restrictions. 
Rehabilitation interventions should simultaneously have positive benefits on overall athletic performance. Unfortunately, the research investigating the effects of stretching (all forms) on athletic performance, such as power and muscle strength, is conflicting. ${ }^{12,21-26}$ Vertical jump height, believed to be a strong determinant of power, decreased following PNF stretching during a pre-competition warm-ups, while another study found no significant differences in vertical jump following an acute stretching intervention. ${ }^{21,22,23}$ Maximum voluntary isometric contractions (MVIC) of the quadriceps muscle group was unaffected by the use of PNF stretching in indoor soccer players. ${ }^{26}$ However, conflicting research has surfaced presenting decreases in MVIC in the hamstrings following an acute PNF stretching intervention. ${ }^{25}$ Due to the inconclusive evidence and the potentially deleterious effects on athletic performance, more research is needed and this study will also investigate the effects of both PNF stretching protocols on vertical jump height and hamstring force output (strength).

Therefore, the purpose of this study was to determine the effects of clinician assisted PNF stretching, unassisted PNF stretching, and no stretching on measures of hip flexion ROM, hamstring MVIC, and maximum vertical jump height. We hypothesized that both the Assisted and Unassisted groups would experience similar increases in passive hip flexion ROM and those changes would be greater in comparison to the control group. We also hypothesized that there would be no significant difference in the amount of change between groups on measures of hamstring MVIC and maximum vertical jump height. If Unassisted PNF stretching is equally effective as clinician assisted PNF stretching, without negatively impacting performance measures, it could be a time saving technique for clinicians and allow independence in the patient. 


\section{CHAPTER II}

\section{REVIEW OF LITERATURE}

\section{Anatomy}

To understand the mechanisms and predisposing factors behind the prevalence and common reoccurrence of hamstring strains, one must first examine and understand the anatomy of the hamstring muscle group.

\section{Hamstrings}

The hamstring muscles serve as the body's primary movers for knee flexion and hip extension with an extended knee. ${ }^{27}$ The hamstring muscle group is comprised of 3 muscles: the biceps femoris, semimembranosus, and semitendinosus. Located on the posterior thigh, these three muscles cross both the hip and knee joints, making them biarticular. The biceps femoris has two portions, a long head and a short head. The long head originates on the ischial tuberosity of the pubic bone and the sacrotuberous ligament. The long head inserts on the lateral side of the lower leg, specifically on the fibular head and lateral tibial condyle. The tibial nerve innervates the long head of the biceps femoris. The short head originates on the lateral lip of the linea aspera and the upper $2 / 3$ of the supracondylar line of the femur and inserts on the fibular head and lateral tibial condyle. The common peroneal nerve innervates the short head of the biceps femoris, a distal branch of the sciatic nerve. The actions of the biceps femoris are hip extension, hip external rotation, knee flexion, and external rotation of the tibia. The semimembranosus originates on the ischial tuberosity and inserts on the posteromedial portion of the medial tibial condyle. The tibial nerve, a distal branch of the sciatic nerve, innervates the semimembranosus. Its actions are hip extension, hip internal rotation, knee flexion, and internal tibial rotation. The semitendinosus originates on the ischial tuberosity and inserts on the medial portion of the tibial 
flare (pes anserine). The tibial nerve, a distal branch of the sciatic nerve, innervates the semitendinosus. The semitendinosus' actions are hip extension, hip internal rotation, knee flexion, and internal tibial rotation. ${ }^{27}$ The hamstring muscles also play an important role in minimizing the amount of anterior tibial translation on a fixed femur which is key in the prevention of ACL tears. ${ }^{5}$ While these muscles' primary concentric motion is hip extension and knee flexion, they also eccentrically contract to control hip flexion and knee extension primarily during the terminal swing phase of the running gait cycle. ${ }^{5,28}$ A concentric contraction is a contraction of a muscle while the muscle is shortening and an eccentric contraction is the contraction of a muscle while that muscle is lengthening, usually to control the movement of an opposing or antagonist muscle. When considering the biarticular nature of the hamstring muscles, this concentric versus eccentric contraction, specifically eccentric strength, becomes very important in one of the mechanisms of injury regarding hamstring strains. Likewise, the length of all three hamstring muscles, individually and as a muscle group, becomes a significant factor in the common overstretch mechanism. Ultimately, clinicians and researchers alike must understand the anatomy of the hamstring muscle group before understanding injury pathologies and developing prevention and rehabilitation programs to treat those injuries.

\section{Hamstring Strain: What Is It?}

Before discussing how to prevent and treat hamstring strains, one must first understand what a hamstring strain is. A hamstring strain ,symptomatically, is described as posterior thigh pain without the involvement of direct contact to the leg. ${ }^{2,3}$ Turner et al. ${ }^{5}$ describes a muscle strain as a disruption of the musculotendinous unit, usually at the proximal attachment. The most vulnerable portion of a muscle is at the musculotendinous junction where the force generated by the muscle fibers is transmitted to the tendon, which then is transmitted to the bone for 
movement to occur. ${ }^{29}$ In regards to the hamstring muscle as a group, the musculotendinous junction lies near the ischial tuberosity origin and near the individual muscles distal insertions. In particular, the muscle that is most commonly injured is the biceps femoris on the lateral side, accounting for $84 \%$ of hamstring injuries in UEFA elite soccer players and $53 \%$ of hamstring muscle injuries in a similar study. ${ }^{5,30}$

Hamstring strains typically involve a similar mechanism of injury and result in a subsequent "grade" of injury. Hamstring strains are categorized into grades depending on the severity of the injury. Accordingly, each grade involves varying magnitude of tearing of the muscle fibers and resultant functional deficit. A grade 1 is a minor or mild strain involving a minimal tear of the musculotendinous unit and minor loss of strength and function. Secondly, a grade 2 is a moderate strain with a partial tear of the musculotendinous unit and a significant loss of strength resulting in a functional deficit and may be represented by some ecchymosis. Lastly, a grade 3 is a severe injury that involves a complete rupture of the musculotendinous unit and results in severe functional deficits. ${ }^{2}$ This may be represented by severe ecchymosis, an obvious deformity, and complete loss of function. While the grade of the injury is important in the creation, implementation and progression of a rehabilitation program, the mechanism of injury is a very important aspect of the hamstring injury.

Askling et al..$^{31,32}$ has identified the 2 most common types of hamstring strain mechanisms, high-speed running and overstretch mechanisms. High speed running, typically involves the biceps femoris long head tendon. The other, an overstretch injury resulting from a high kick or split, usually involves the semimembranosus muscle. ${ }^{33}$ These overstretch injuries typically involve the proximal free tendon of the semimembranosus muscle and may occur more proximal to the ischial tuberosity of the pelvic bone. ${ }^{31,32}$ Research regarding overstretch injuries 
has primarily focused on the dancer population, who are more commonly exposed to highkicking or sagittal split maneuvers. ${ }^{31,32,34}$ Further research by Askling et al. ${ }^{32,35}$ supported the notion that a majority of high-speed running injuries occur in the biceps femoris as all of the sprinters' primary injuries involved solely the biceps femoris muscle. The rapid acceleration mechanism most commonly results from a rapid change to knee flexion and hip extension following hip flexion and knee extension in high speed running. ${ }^{3,36}$ A combination of hip flexion and knee extension places the biarticular hamstring muscle in its' most lengthened position, largely contributing to the rate of injury during high-speed movements. ${ }^{5,37}$ Another aspect of this common mechanism is the rapid rate of change from hip flexion and knee extension to hip extension and knee flexion near the terminal swing and ground contact phases of the running gait. ${ }^{1,38,39}$ This is the crossover of maximum eccentric contraction to the maximum concentric contraction in which the hamstring, specifically the biceps femoris, is most vulnerable to an eccentric overload injury. ${ }^{1,36,40,41}$

One proposed idea to why the biceps femoris is most commonly injured during high speed running is that this muscle is at its greatest stretch near the ground contact phase of running. Various reasons have been discussed regarding this idea, from the biceps femoris being the longest of the three hamstring muscles, to the two different innervations of the long and short heads of the biceps femoris. ${ }^{5,29}$ Yet, it has been shown that hamstring injuries involving an overstretch mechanism take longer for athletes to return to play compared to high-speed running injuries. ${ }^{32,34}$ An important observation is that of the information provided, both injury mechanisms can be applied to sport-related soccer specific hamstring injuries. Soccer players are frequently sprinting throughout training sessions and matches as well as completing slide tackles, high kicks, and stretching to reach passes. ${ }^{1}$ These studies and results can help clinicians identify 
the most influential risk factors and implement techniques to help target and modify these factors in an attempt to decrease the frequency of hamstring strains in elite soccer players.

Risk factors that contribute to hamstring strains can be categorized as modifiable or nonmodifiable as well as intrinsic or extrinsic. ${ }^{1,5}$ Examples of modifiable risk factors include the flexibility of the quadriceps muscles and hamstring muscles, muscle imbalances, fatigue, and strength, all of which can be altered or modified using various training and sport performance techniques. ${ }^{5}$ Non-modifiable factors include factors that cannot be changed such as age, previous injuries, and ethnicity. ${ }^{5}$

Extrinsic risk factors are those outside of the body, including environment-related factors such as playing surface or a poor warm-up. Intrinsic risk factors are player-related factors including muscle weakness, instability, fatigue, psychosocial factors and flexibility. ${ }^{3}$ The one modifiable, intrinsic factor that this study will focus on is flexibility. Flexibility is a vital component of a physical conditioning program that allows the tissue to easily accommodate to stress, dissipate shock impact, and improve efficiency of movement through a decreased resistance of soft-tissue structures surrounding the joint, thus minimizing or preventing injury. ${ }^{6,9,42,43}$ Simply, flexibility is the ability for a joint to move and bend through a full range of motion. ${ }^{9}$ The most widely utilized method of increasing muscle flexibility is through stretching techniques. $^{6}$

Unfortunately, recurrent hamstring strains are a large concern in soccer. One research study reported $1 / 3^{\text {rd }}$ of hamstring injuries possess the greatest risk of recurring within 2 months of the initial return to activity. ${ }^{36,44}$ Likewise, a study by Petersen et al. ${ }^{45,46}$ found that $25 \%$ of Danish professional soccer players with previous hamstring injuries were likely to sustain a recurrent injury the following season. Previous injury has been shown to be a significant factor in 
the likelihood of hamstring re-injury, but often is one component of a multitude of factors. ${ }^{1,47}$ Other factors include an insufficient rehabilitation program, return to full participation too soon, or a combination of the two. The relatively high rate of re-injury can also be attributed to decreased extensibility due to scar tissue, persistent weakness of the injured muscle, and biomechanical and motor pattern adaptations following the original injury. Despite the various injurious factors, the rehabilitation program can be the most important aspect of returning from injury while keeping focus on the modifiable factors to try and return the athlete to their respective sport at a high performance level with minimal risk for recurring injury. ${ }^{36}$ Previous research has demonstrated that incorporating eccentric hamstring strengthening and closely monitoring return to activity decision making can lead to a reduction in the recurrent rate of hamstring injuries. ${ }^{36,48}$ While this method is trusted and supported by literature, one aspect that must be considered is the incorporation of a flexibility program into an athletes' prevention and rehabilitation program. ${ }^{5}$ While results are conflicting on flexibility's influence on the prevention of primary and recurrent hamstring injuries, the focus of our study will investigate the effects of stretching on flexibility and athletic performance measures.

\section{Stretching Mechanism}

Stretching is the most widely used method for increasing flexibility and has been shown to increase ROM, benefit athletic performance, and increase neuromuscular efficiency in some cases. ${ }^{6,7}$ A study by Wicke et al. ${ }^{9,49}$ stated that stretching exercises increased coordination by improving the nerve impulse velocity that allows the muscles to coordinate better by recruiting muscle fibers that previously failed to contract. Furthermore, Shrier ${ }^{42}$ found that possessing an appropriate amount of flexibility can lead to reduced injury risk and improved performance by decreasing the resistance of the tissue surrounding the respective joint. This allows the joint and 
the athlete to work and move more efficiently. ${ }^{9,42}$ To better understand how stretching works, there needs to be an explanation and understanding of the proposed mechanisms of stretching. There are two types of mechanoreceptors in the body that are important for promoting muscle relaxation and the stretch reflex. These mechanoreceptors are muscle spindles and Golgi tendon organs (GTO). ${ }^{7,8,10,12,47}$ Muscle spindles and golgi tendon organs (GTO's) are mechanoreceptors sensitive to changes in muscle length and tension, respectively. Muscle spindles are housed in the muscle belly and provide afferent sensory signals to the brain and spinal cord to encourage a reflexive resistance to stretch through muscle activation. Prentice et al. ${ }^{47}$ states that while a muscle is being stretched, the muscle spindles and GTO's are simultaneously sending afferent signals to the brain that the muscle is being stretched. The muscle spindles signal stimulates the spinal cord, which in turn sends efferent signals back to the muscle causing the muscle to reflexively contract as a protective mechanism. ${ }^{8,47,50}$ If the stretch is held for at least 6 seconds afferent signals from the GTO's begin to override the muscle spindle efferent signals. GTO's are protective mechanoreceptors located in the tendon that provide information to the brain in response to excessive tension in a muscle due to stretch or forceful contraction. ${ }^{12,47}$ GTO's are different from muscle spindles in that they encourage a reflexive relaxation to the muscle being stretched. As the GTO's afferent signals are sent to the brain, they override the muscle spindle signal and lead to the activation of inhibitory interneurons within the spinal cord. These interneurons send an inhibitory stimulus through the alpha motorneuron, decreasing the nerves excitability and decreasing the muscles efferent motor drive. This ultimately leads to relaxation of the muscle being stretched, which can lead to an increase in the range of motion of a joint. ${ }^{8,12,47}$ The "stretch reflex" consists of the muscle spindle producing a resistive reflex to stretch while the GTO produces a relaxing reflex of the muscle in response to a stretch. ${ }^{47}$ 
There are a number of stretching techniques utilized in the clinical and research settings; including static, dynamic, ballistic, and PNF stretching. ${ }^{5-11}$ Static stretching is a technique that has long been used to help increase muscle flexibility, and it is one of the most frequently used stretching techniques. ${ }^{7}$ Static stretching involves stretching a muscle to its end range and holding that stretch for a period of time with a sustained amount of force.$^{51}$ Dynamic stretching involves actively moving joints through a full range of motion and is often used in a pre-exercise warmup. ${ }^{9}$ Ballistic stretching involves a bouncing movement in which repetitive contractions of the agonist muscle are used to produce quick stretches of the antagonist muscle. ${ }^{47}$ Ballistic stretching has faced some criticism because the potential increase in range of motion comes at the risk of injury. ${ }^{47,52} \mathrm{PNF}$ stretching increases joint range of motion by incorporating passive stretches with a variety of voluntary muscle contractions (isometric, isotonic) to override the sensorimotor system and promote muscle relaxation. ${ }^{7}$ Hindle et al. ${ }^{12}$ support the notion that PNF stretching increases range of motion by increasing the length of the muscle and the neuromuscular efficiency of the muscle. Rowlands ${ }^{8,16}$ found that over a 6 week time period, PNF stretching significantly increased range of motion by 32 degrees using a contract-relax-antagonist-contract PNF stretching technique. Previous research supports the use of PNF stretching to improve range of motion, and in some cases, more beneficial than static stretching. ${ }^{8,10,13,15,53-57}$ A more in depth explanation of PNF stretching follows.

\section{Proprioceptive Neuromuscular Facilitation: PNF}

Proprioceptive neuromuscular facilitation, better known as PNF, is a stretching technique utilized to improve muscle elasticity and has been shown to have a positive effect on active and passive ranges of motion. ${ }^{12-14} \mathrm{PNF}$ has been utilized by therapists to restore function to patients who sustained soft-tissue injuries and even invasive surgeries. ${ }^{8}$ Many elements combine to create 
a PNF protocol: agonist and/or antagonist muscle contraction, duration of contraction phase, duration of relaxation phase, ratio of contraction to relaxation durations, contraction intensity, number of contractions, and timing of movement from end of contraction to new point of bind. ${ }^{58}$ PNF stretching manipulates the sensorimotor system through a variety of mechanisms, including autogenic inhibition, reciprocal inhibition, stress relaxation, and the gate-control theory.

There are two proposed mechanisms underlying the PNF stretching response: autogenic inhibition and reciprocal inhibition. Autogenic inhibition is described as a reflexive activation of the muscle that is experiencing a stretch or forceful contraction (tension) and is due to the inhibitory effect of the GTO in the tendon. ${ }^{8,47,59}$ The contract-relax PNF stretching technique incorporates an antagonist, muscle being stretched, isotonic muscle contraction which increases the tension sensed by the GTO and a subsequent reflexive inhibition. ${ }^{60}$ Autogenic inhibition overrides the myotatic reflex and leads to decreased neural activity, which leads to potential lengthening of the target muscle. ${ }^{8,16}$

Reciprocal inhibition occurs when a contraction in one muscle (agonist) signals inhibition in the opposing (antagonistic) muscle. ${ }^{8,12}$ This relaxation of the target muscle prevents the agonist and antagonist muscles from working against each other and could allow for an increase in stretch resulting in an increased range of motion.

Other potential mechanisms behind PNF's effectiveness are mechanical changes within the musculotendinous unit itself and an overall altered perception of stretch by the individual resulting in an increased stretch tolerance. ${ }^{24}$ Other influential factors can include age, gender, muscle, stretch duration, PNF technique, contraction duration, and percentage of maximum voluntary isometric contraction utilized. ${ }^{12,16,20,61}$ 


\section{PNF Stretching Techniques}

Three PNF stretching techniques will be described in greater detail, 1) hold-relax, 2) slow-reversal-hold-relax, and 3) contract-relax. ${ }^{6,12,17,18}$ Overall, PNF stretching techniques are based on optimizing relaxation of the muscles which leads to a greater stretch. ${ }^{16}$ For consistency in discussing PNF stretching throughout the remaining review, we will "antagonist" to describe the muscle being stretched and "agonist" to describe the opposing muscle(s). ${ }^{8}$ Specifically for this study, the hamstring muscles are the antagonist or target muscle receiving the stretch and the quadriceps and hip flexors are the opposing or agonist muscle groups.

The Hold-Relax PNF technique involves passively moving the body part into the agonist movement pattern, placing a stretch on the antagonist or target muscle. This is immediately followed by an isometric contraction of the antagonist muscle against resistance for at least 6seconds. Finally, a concentric contraction of the agonist muscles brings the limb into a position

to once again place a maximal stretch on the antagonist muscle group. ${ }^{18,51}$ This technique is used when muscle tension is present on one side of the joint. The Slow-Reversal-Hold-Relax PNF technique involves passively moving the body part into the agonist movement pattern, placing a stretch on the antagonist or target muscle. This is immediately followed by an isotonic muscle contraction of the agonist (quadriceps) followed by an isometric contraction of the antagonist (hamstrings, target muscle) during the push phase. Next, the antagonist muscle is relaxed while the agonist contracts once again causing movement through the new range of motion. This technique is useful when the limiting factor in range of motion is the antagonistic muscle group. $^{18}$

The Contract-Relax PNF technique also involves passively moving the body part into the agonist movement pattern, placing a stretch on the antagonist or target muscle. This technique is 
followed by an isotonic muscle contraction of the antagonist against the resistance of the clinician moving their limb through a range of motion. Next, the patient relaxes and the antagonist muscle is again passively stretched to a new end range at a point of restriction. This method is most beneficial when muscle tightness limits range of motion. ${ }^{19}$ A study conducted by Yuktasir and Kaya ${ }^{62}$ found that the contract-relax method of PNF stretching increased hip flexion range of motion of 9 subjects after a 6 week study incorporating four PNF contract-relax stretch interventions per week.

For the purposes of the current study we have chosen to utilize the contract-relax PNF technique based on its effectiveness in previous research in eliciting gains in range of motion and because it is indicated for use when range of motion is limited by muscle tightness. ${ }^{63}$ The population investigated in this study will have hamstring muscle tightness. We aim to investigate the effectiveness of the standard technique utilizing a clinician versus performing the same technique with a stretching strap.

PNF stretching has most commonly been performed with a trained clinician or partner. ${ }^{6,910,17,22,24,51,62,64}$ However, other effective methods of applying resistance may be beneficial to the clinician and athlete/patient. Three methods for applying manual resistance are by oneself (unassisted), clinician (partner) assisted, or by a fixed object such as the floor or wall. ${ }^{9}$ Maddigan et al. ${ }^{10}$ utilized a Theraband stretch strap as a means of resistance and to conduct passive movement to a new end range or point of limitation. Similar to partner-assisted PNF stretching, the use of a stretch strap allowed subjects to complete concentric hamstring contractions as well as passively stretch to a new point of resistance. ${ }^{10}$ Ultimately, PNF stretching has shown promise in regards to range of motion, yet we must also consider and research the effects of PNF stretching on athletic performance measures. Muscle force through 
the measure of maximum voluntary isometric contractions (MVIC) and maximum vertical jump height have been debated in the literature in regards to the effects that stretching may have on them.

\section{Effects on Maximum Voluntary Contraction}

MVIC is a standardized, objective and sensitive tool for the measurement of muscle strength. MVIC can be measured using a hand held dynamometer (strain gauge), which quantifies the force output by the patient in Newtons. This type of measurement is preferred over clinical manual muscle testing (MMT) because it is objective and it does not use a generalized grading scale. MVIC has also been shown to be more reliable than MMT. ${ }^{65}$ Research has demonstrated no significant increase or decrease of MVC following PNF stretching of the rectus femoris and vastus lateralis muscles following an acute PNF stretching intervention, ${ }^{26}$ which is conflicting with the deficits of PNF and static stretching on MVC of the biceps femoris. ${ }^{25}$ The conflicting results of these studies leaves room for further research and an opportunity to compare the effects of self-directed and partner assisted PNF stretching on MVIC of the hamstring muscles.

\section{Effects on Vertical Jump Height}

Maximum vertical jump height is a measure of how high an individual or athlete can elevate off the ground from a standing position. It is believed to be a strong indicator of athletic performance and is often used in the clinical and research setting. Church et al. ${ }^{21}$ investigated the effects of static stretching versus PNF stretching during a warm-up on measures of vertical jump height. The warm-up that incorporated a PNF stretch displayed a decrease in vertical jump height. However, similar research has found no effects of PNF stretching on vertical jump height. $^{24,22,23}$ Due to the inconclusive evidence, we feel it is important to also investigate the 
effects of self-directed and clinician-assisted PNF stretching over the course of 6-weeks on measures of maximum vertical jump height.

\section{Clinician-Assisted versus Self-Directed PNF Stretching}

In several of the studies that involved a PNF stretch intervention, there was mention of partner assistance with a clinician or therapist. ${ }^{6,7,10,23,25,26,51}$ On the other hand, there has been increase in the number of studies that involve a PNF intervention with the resistance provided by either an object or the subjects themselves. ${ }^{9,17,24,58,66}$ This has created a new idea that PNF stretching may no longer require the need of a partner, as self-directed PNF stretching can be just as effective. The use of self-directed PNF stretching has been supported as a more effective tool for increasing flexibility versus the use of static stretching. ${ }^{9,57,62,67}$ Schuback et al. ${ }^{17}$ found a significant increase in hip flexion range of motion when utilizing a self-stretch PNF technique as well as a clinician-assisted PNF technique. Both techniques resulted in significant increases in range of motion of 9.6 degrees and 12.6, respectively. Studies by Wicke et al. ${ }^{9}$ and Place et al. ${ }^{24}$ have provided evidence that PNF stretching can be performed safely and effectively without the need for a partner. Wicke et al. ${ }^{9}$ utilized a chair as the means of resistance in a twelve-week crossover intervention. Subjects completed both a static stretching intervention and a PNF stretch intervention for six-weeks a piece which both resulted in increases in sit-and-reach measures and hip range of motion specific to the PNF intervention. Place et al. ${ }^{24}$ utilized a box as a means of resistance during an acute, 7-day PMF stretch intervention. While no significant increases resulted, neither did any deficits in range of motion, countermovement jump, or squat jump. Most significant is one study that compared assisted versus unassisted PNF stretching versus static stretching introduced the use of a stretch strap as a method of resistance during the contraction phases of PNF stretching routines. ${ }^{10}$ This study is significant in being the only study 
that utilized a stretch strap as a tool to complete an unassisted PNF stretching protocol. The unassisted PNF technique yielded similar hip flexion range of motion increases as the static and partner-assisted PNF stretching techniques. Furthermore, all three techniques showed similar deficits on movement time and dynamic angular velocity. The introduction of the stretch strap could prove significant in assisted PNF stretching versus a box or chair. Coupled with benefits on hip range of motion and inconclusive results on athletic measures, there is an obvious need for further research comparing partner-assisted PNF stretching versus unassisted PNF stretching by way of a stretch strap. The use of self-directed PNF stretching eliminates 2 elements that partner-assisted PNF requires: the need for a partner and the time commitment of both parties when both parties need to be stretched. ${ }^{9}$ By eliminating the need for a partner, the time commitment is decreased as well as control of the stretch and all associated factors is in control of the person that is stretching. Further research is needed to not only compare clinician-assisted and self-directed PNF stretching but also to compare their respective effects on athletic performance measures. There has been support of self-directed PNF stretching over partnerassisted in regards to convenience and time commitment yet the decision on which technique is better regarding flexibility and influence on performance remains inconclusive. ${ }^{6,7,9,10,24-26}$ This leaves room for the comparison of unassisted and clinician-assisted PNF stretching on the aforementioned variables of MVIC, vertical jump height, and range of motion. 


\section{CHAPTER III}

\section{METHODS}

\section{Research Design}

This study used a cross-sectional 2-group pre-test / post-test design to investigate the effectiveness of two variations of PNF stretching on hamstring flexibility and athletic performance. The independent variable was group with 3 levels (Unassisted PNF Stretching, Assisted PNF Stretching, Control). The pre-and post-test measures included passive hip flexion range of motion (ROM), hamstring maximum voluntary isometric contraction (MVIC) which was normalized to the participants' body weight, and maximum vertical jump height. Normalizing the MVIC measurement to the participant's body weight helped minimize the effect that body mass may have on the isometric force output measurement. The dependent variables of interest were change scores (Post - Pre) calculated for each measurement. The investigator (JTL) was blinded to ROM and MVIC measures but was not blinded to group allocation as he supervised all training sessions.

\section{Participants}

A total of 26 healthy, college aged, individuals with hamstring tightness participated in this study (age $=20.12 \pm 2.91 \mathrm{yrs}$, height $=172.69 \pm 6.72 \mathrm{~cm}$, mass $=70.50 \pm 14.05 \mathrm{~kg})$. The inclusion criteria were healthy, physically active, adults between the ages of 18-30 years that demonstrated hamstring tightness measured through a passive straight leg raise (SLR) resulting in a hip flexion angle of $\leq 90^{\circ}$ on the dominant kicking limb. Measures were recorded bilaterally because the stretching intervention was performed bilaterally, but only the dominant limb measures were used for data analysis. $\left(<90^{\circ}\right)$. Physically active was defined as participating in moderate level physical activity 3 times per week for 20 minutes or more each session. ${ }^{68}$ 
Participants were excluded if they had a hamstring injury in the past 6 months, any lower extremity injury that kept them out from physical activity for more than 2 days, a history of lower extremity surgery, neurological disorders, or recent participation in a lower extremity stretching program (past 6-weeks).

\section{Instrumentation}

A digital inclinometer (Pro 3600 Digital Inclinometer; SPI-Tronic, Garden Grove, CA) was used to measure hip flexion angle during the passive SLR. The PRO 3600 Digital Protractor is a revolutionary measuring tool that provides an immediate, digital reading of all angles in a $360^{\circ}$ range. Intra-rater reliability for this measurement was established by the investigator (JTL) prior to data collection. $\left(\operatorname{ICC}_{(3, \mathrm{k})}=0.99 ; \mathrm{SEM}=3.53^{\circ}\right)$ and between-day reliability $\left(\mathrm{ICC}_{(3, \mathrm{k})}\right.$ $\left.=0.93 ; \mathrm{SEM}=5.53^{\circ}\right)$

Maximum voluntary isometric contraction (MVIC) is used as a tool to help gauge the amount of force that a muscle or muscle group is producing through a $100 \%$ intensity isometric contraction held for 5-seconds. A handheld dynamometer (Lafayette Manual Muscle Testing System Model 01163 Lafayette Instruments, Lafayette, IN) was used for the measurement of hamstring MVIC. The test was performed with the clinician applying matching resistance to the limb of the participant against their maximal muscle isometric contraction. Peak force in kilograms $(\mathrm{kg})$ was recorded for each of the three trials and later converted into Newtons (N) for further data analysis. Intra-rater reliability was established for the use of hand-held dynamometry prior to data collection $\left(\operatorname{ICC}_{(3, \mathrm{k})}=0.91 ; \mathrm{SEM}=1.67 \mathrm{~N}\right)$ and between-day reliability $(\operatorname{ICC}(3, \mathrm{k})=$ $0.90 ; \mathrm{SEM}=1.22 \mathrm{~N})$.

Maximal vertical jump height was assessed using the Vertec Vertical Jump Height Testing System (Vertec Vertical Jump Measuring Device; Gill Athletics, Champaign, IL). The 
vertical jump demonstrates athletic ability based on the measure of their explosive strength and their vertical jump.

The Unassisted stretching protocol was implemented using the Dynamic Stretch Strap

(Power Systems, Knoxville, TN), which makes it possible to stretch muscles that are difficult to reach through unassisted stretches without the need for a clinician. This strap was used as an unassisted, passive stretching tool as well as a means for resistance with the Unassisted PNF stretching protocol. The strap is a durable, nylon strap ( 75 1/2” L x 1" W) with 10 elastic loops that can be used to pull muscles through a full range of motion.

\section{Procedures}

\section{Consenting Process \& Demographics}

Participants were recruited using IRB approved fliers, television advertisements, emails, and classroom visits. All potential participants reported to the data collection site wearing their own comfortable athletic shorts, athletic t-shirt, and athletic shoes. Upon arrival, the investigator (JTL) gave a brief explanation of the nature of the study and participants read and signed an IRB approved consent form (Appendix I). Demographic information was recorded, including age, sex, height $(\mathrm{cm})$, body mass $(\mathrm{kg})$, and dominant leg. All participants also completed a health history questionnaire (Appendix II) to gather information on previous injury history and physical activity level. Individuals that reported previous lower extremity injury in the last 6 months, history of lower extremity surgery, or neurological disorders were thanked and excluded from the study $(\mathrm{n}=2)$. At the completion of participant consent and demographic information collection, the screening process for passive hip flexion ROM was completed. 


\section{Screening Procedure: Passive Hip Flexion ROM}

Screening for inclusion in this study included a measurement for hamstring flexibility using a passive SLR, or hip flexion range of motion measurement with the knee extended. Prior to range of motion screening, all participants performed a 5-minute warm-up on a stationary bike. Each participant was asked to lie on their back, in a supine position, with the non-test hip in a neutral position and stabilized to the table by the research assistant (BH/MK). The digital inclinometer was zeroed relative to the horizontal and then was positioned along the midline of the femur at the distal point of the greater trochanter (Figure 1). The test hip was passively flexed, while the knee was stabilized in full extension (Figure 1). End range of motion for hip flexion was determined at a point of resistance felt and determined by the research assistant (BH/MK). Once the end ROM was found, the investigator (JTL) pressed the hold button on the digital inclinometer and presented the screen with the value to the research assistant to record. This process was completed three times on each leg with 30 -seconds rest in between trials. The mean of the three values of the dominant leg was used for statistical analysis. Inclusion criteria was met if all 3-trials resulted in a hip flexion angle of $\leq 90^{\circ}$ bilaterally. This finding was indicative of hamstring/posterior thigh tightness and these individuals were included in the study. Any participants with a hip flexion angle $>90^{\circ}$ with respect to either leg were excluded from the study at this time $(\mathrm{n}=0)$.

Potential participants who fit all criteria to participate in this study were then randomly assigned to one of three groups; the Unassisted PNF, Assisted PNF, or Control group. Participants selected a piece of paper from an opaque envelope, which assigned them to one of the three groups. Males and females picked from separate envelopes to ensure equal numbers of male and female participants were allocated to each respective intervention or control group. At 
the conclusion of group allocation, all participants completed pre-test measurements for hamstring MVIC and maximal vertical jump height.

\section{Pre-Test Measurements}

The hip flexion ROM measurements recorded during the screening process were used as the pre-test measure.

Hamstring MVIC measurements were performed with the participants lying prone on a treatment table. ${ }^{28}$ The knee of the test leg was flexed to 90 degrees while the other leg remained in a neutral position on the table. The investigator stood behind the table on the same side as the test leg (Figure 2). The handheld dynamometer was placed on the posterior surface of the lower leg 3-centimeters proximal to the lateral malleolus. This measurement was similar to the position of the collar used for placement of a strain-gauge in a previous study that tested MVIC of the hamstring muscles. ${ }^{69}$ The participant was instructed to perform a maximal isometric hamstring muscle contraction for a total of 5-seconds, pulling their heel in toward their buttocks against the resistance of the examiner, who attempted to match the resistance of the contraction for the most accurate strength measurement. The investigator encouraged maximal effort by providing verbal encouragement. A research assistant read and recorded all peak force measures in kilograms (kg), which allowed the investigator (JTL) to remain blinded to these measurements. The values in kilograms $(\mathrm{kg})$ were later converted to Newtons $(\mathrm{N})$ by the research assistant. Three 5 -second trials were performed with a 60 -second rest period in between each trial. ${ }^{70}$

Maximum vertical jump height measurements began with the participant instructed to stand underneath the Vertec system with one arm raised as high as possible over their head. The Vertec was adjusted so that the lowest peg touched the tallest point on their hand. The participant then completed a counter movement jump for maximal height and attempted to hit the highest 
veins. To perform the countermovement jump, participants were instructed to start standing upright with their feet shoulder width apart and arms relaxed by their sides with elbows flexed to 90 degrees. When instructed to, the participants completed a rapid countermovement by squatting and flexing the knees to about 90 degrees while allowing the shoulders and elbows to swing into extension. Once optimal flexion is reached, the vertical jump began with an explosive extension of the legs in an attempt to jump and achieve the highest vertical height possible. The participant reached and extended the elbow as far as possible trying to reach the maximum height on the Vertec Jump Height Measuring System. ${ }^{71}$ The space between each peg represents $1 / 2$ inch and researchers multiplied the number of pegs moved every $1 / 2$ inch to calculate maximum vertical jump height. Three trials were performed with 30 -seconds of rest in between. The mean of three trials was used for data analysis.

The order or pre-test measurements was standardized, identical across participants.

\section{Intervention}

Regardless of stretching intervention group, all participants completed the stretching protocol 12 times over a 6-week time period (twice per week). Previous research has demonstrated that a 6-week hamstring PNF stretch interventions resulted in significant ROM increases. ${ }^{16,72}$ All sessions were performed in the testing facility. All participants were asked to not change anything with their exercise routine and report to the data collection site 7-weeks later for post-test measures.

Participants performed a 5-minute warm-up on a stationary bike prior to each stretching intervention session. Both PNF stretching groups utilized the Contract-Relax PNF stretching technique. The technique is fully described here and the variations are described under the group intervention. The contract-relax PNF technique utilizes a passive stretch of the hamstring 
followed by an isotonic muscle contraction of the hamstring and an additional passive stretch. The initial stretch of the hamstrings was performed passively by moving the leg into hip flexion with the knee fully extended to the point of first restriction. This passive stretch was held for 10seconds and immediately followed by an active concentric, isotonic contraction of the hamstrings moving into hip extension for 7-seconds. At the completion of 7-seconds, the participant relaxed and an additional passive stretch of the hamstrings with the knee fully extended was performed to the new point of restriction for a total of 10 -seconds. ${ }^{73}$ Following the second 10-second stretch, the leg was lowered back to the table and rested for 10 seconds after which the procedure was repeated. A total of three sets of the entire process were performed on each leg with a 10 -second rest period in between each repetition.

The Unassisted PNF stretching group (n=9; Age $20.00 \pm 1.50$; Height $(\mathrm{cm}) 173.22 \pm$ 3.15; Mass (kg) 67.19 \pm 4.81 ) utilized arm strength and a Dynamic Stretch Strap (Power Systems, Knoxville, TN) to provide the passive stretch and resistance components of the PNF stretching technique. For each session, participants reported to the testing facility and signed in on a check-in sheet so that the investigator (JTL) could track the number of sessions completed. Participants followed printed "Unassisted PNF stretching" protocol flyers, which were posted at the data collection sites, which instructed participants on how to complete the stretch intervention (Appendix III). In addition to the descriptive flyers, participants used the MetroTimer App that was downloaded to their smartphone to help track time and guide them to correctly complete the timed portions of the stretch protocol. This protocol instructed participants to keep their leg relaxed (knee extended) while using their arm strength to pull on the stretch strap and move their leg into passive hip flexion until the point of restriction and held for 10 -seconds. The participants then contracted their hamstrings in an isotonic contraction 
moving their leg back down toward the table while providing resistance with their arm strength with the stretch strap. (Figure 3). This contraction utilized the participant's maximal effort. The participant relaxed the hamstring muscles and performed another 10 -second passive stretch by using their arms and the stretch strap to pull the leg into a stretch. After, the leg was lowered to the table and rested for 10 seconds. The same procedure was completed three times on each leg. A verbal instruction script was used and posted at the data collection sites to confirm consistency in instructions across participants.

The Assisted PNF stretching group (n=7; Age 19.57 \pm 1.51 ; Height $(\mathrm{cm}) 173.43 \pm 6.88$; Mass (kg) $72.10 \pm 12.40$ ) had the investigator (JTL), lead and perform all stretching sessions. The clinician provided a passive stretch to the hamstring to a point of first restriction and held the stretch for 10-seconds (Figure 4) with the aid of the MetroTimer App that was downloaded to his (JTL) smartphone to help track time and guide to complete the timed portions of the stretch protocol correctly. The clinician instructed the participant to immediately follow the passive stretch by contracting the hamstring muscles and pushing down against resistance towards the table while the investigator allowed the leg to lower back down to the table. This was followed by the participant relaxing the leg and another 10 -second passive hamstring stretch to a new point of restriction. Lastly, the leg was lowered back down to the table and rested for 10 seconds. The same procedures were completed for three repetitions per leg with 10-seconds of rest in between.

The control group $(\mathrm{n}=10$ Age $20.60 \pm 2.01$; Height $(\mathrm{cm}) 171.70 \pm 9.13$; Mass $(\mathrm{kg}) 72.36$ $\pm 20.20)$ did not perform a PNF stretching intervention. They were asked to maintain their current physical activity routine and not add any additional modes of exercise. They returned to the research laboratory after 6-weeks for post-test measurements. 


\section{Post-Test Measurements}

All post-test measurements were completed in an identical manner to previously described in pre-test measurements. All post-test measures were taken within one-week of completing the last stretching-intervention session or in week 7 for control participants. The participants in the control group did not complete any stretch intervention and only returned after a 6-week period to complete post-test measurements.

\section{Data Reduction}

Peak force data during the hamstring MVIC was normalized so that comparisons between individuals could be made. The arithmetic mean of three trials was calculated and normalized using an allometric scaling method based on the principle of geometric similarity. ${ }^{74,75}$

Specifically, dividing strength by the body size variable raised to an appropriate power has been theorized to eliminate the effects of body size. This procedure normalizes force measured by the dynamometer and takes into account variations in muscle cross-sectional area as a function of

body mass $(\mathrm{kg})$. The equation used for normalization in this study was $S_{n}=S / m^{2 / 3}$, where $S_{n}$ is the normalized strength value, $\mathrm{S}$ is the force $(\mathrm{N})$ measured by the hand-held dynamometer, and $\mathrm{m}$ is the body mass $(\mathrm{kg})$.

There were three dependent variables of interest for this study; Passive hip flexion ROM change, hamstring MVIC change, and maximum vertical jump change. Change scores were calculated using the arithmetic mean of 3-trials for all three measurements by subtracting the average pre-test value from the average post-test value (Post - Pre).

\section{Statistical Analysis}

Statistical analysis was performed using SPSS (IBM SPSS Statistics for Windows, version 21.0; IBM Corp, Armonk, NY). Separate One-way ANOVA's were performed for each 
dependent variable (ROM Change, MVIC Change, VJ Change) to identify differences between groups (Control, Assisted, Unassisted) in the amount of change that occurred over the course of the 6-weeks. Post-hoc testing was performed utilizing Tukey's LSD to identify the location of differences. Alpha was set a priori at $\mathrm{p} \leq 0.05$. 
Figure 1. Placement of Digital Inclinometer

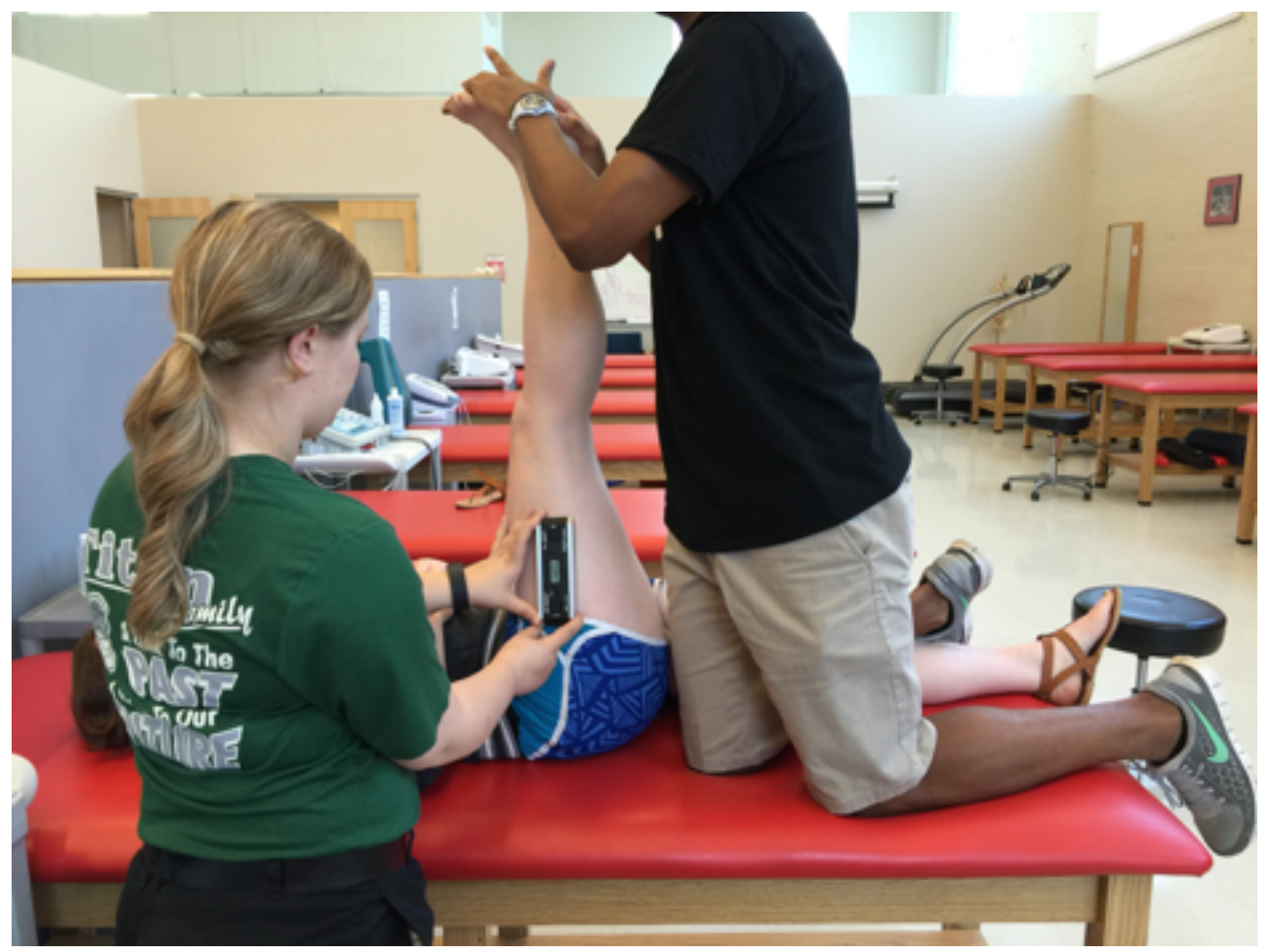

Figure 2. Placement of Handheld Dynamometer

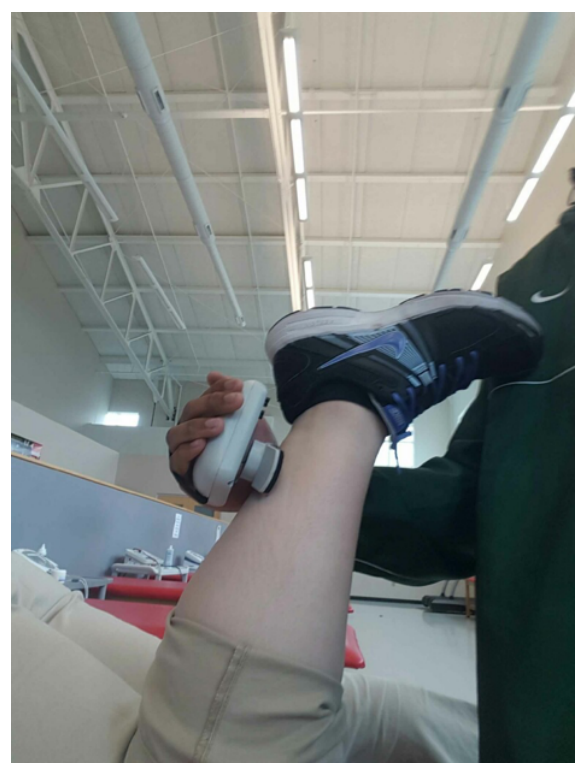


Figure 3. Unassisted PNF Stretch Protocol

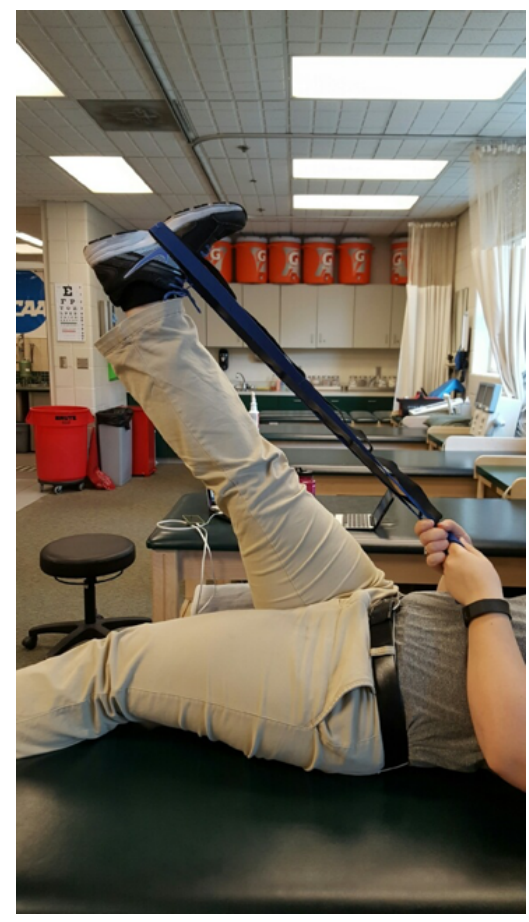

Figure 4. Assisted PNF Stretch Protocol

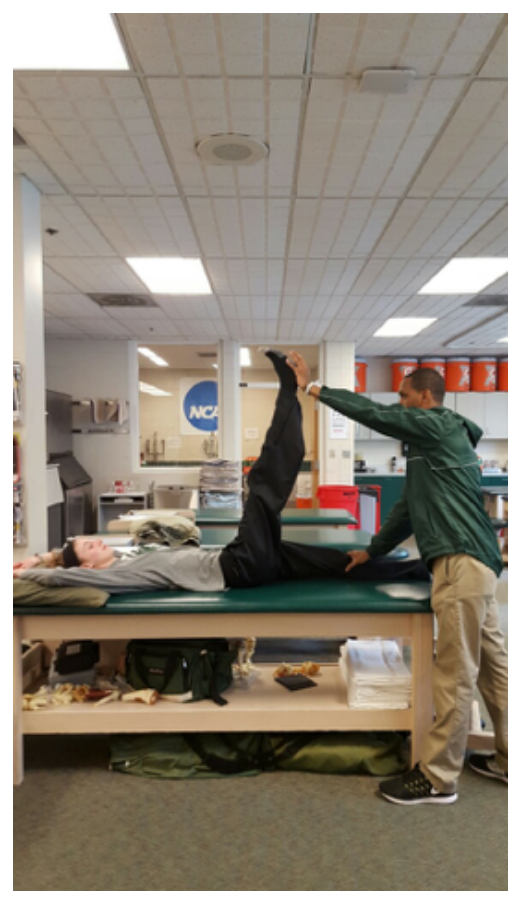




\section{CHAPTER IV}

\section{RESULTS}

The one-way ANOVA for hip flexion ROM was significant $\left(\mathrm{F}_{(2,25)}=9.853, \mathrm{p}=0.001\right)$, indicating there were significant differences in the amount of change in ROM when compared across groups. Post-hoc comparisons found both the Unassisted $\left(10.26^{\circ} \pm 6.03, p=0.002\right)$ and Assisted $\left(10.27^{\circ} \pm 3.81, \mathrm{p}=0.004\right) \mathrm{PNF}$ intervention groups had a greater improvement in $\mathrm{ROM}$ compared to the Control group $\left(-0.41^{\circ} \pm 7.00\right)$. There were no differences between the amount of change in the Unassisted group compared to the Assisted group.

No significant differences were observed when comparing change scores across groups on measures of hamstring MVIC $\left(\mathrm{F}_{(2,25)}=2.542, \mathrm{p}=0.101\right)$ or maximum $\mathrm{VJ}\left(\mathrm{F}_{(2,25)}=0.133, \mathrm{p}\right.$ $=0.877)$. Descriptive data for change scores with standard deviations are presented in Table 1 .

\section{TABLE 1}

Magnitude of Change for Dependent Variables

\begin{tabular}{|l|c|c|c|}
\hline & ROM Change $\left(^{\circ}\right)$ & MVIC Change (N) & VJ Height Change (in) \\
\hline Control $(\mathrm{n}=10)$ & $-0.41 \pm 7.00$ & $-0.17 \pm 1.09$ & $1.00 \pm 2.23$ \\
\hline Assisted $(\mathrm{n}=7)$ & $10.27 \pm 3.81^{*}$ & $-0.80 \pm 0.92$ & $0.41 \pm 3.09$ \\
\hline Unassisted $(\mathrm{n}=9)$ & $10.26 \pm 6.03^{*}$ & $0.29 \pm 0.83$ & $0.81 \pm 2.39$ \\
\hline
\end{tabular}

* Indicates a significant difference in the amount of change compared to the control group 


\section{CHAPTER V}

\section{DISCUSSION}

The objective of the present study was to investigate the effects of Unassisted and Assisted PNF stretching on hip flexion range of motion, MVIC, and vertical jump height. A key finding in this study that confirmed our first hypothesis was that both the Unassisted and Assisted contract-relax PNF stretching protocols resulted in significant improvements in hip flexion range of motion compared to the control group. On average, there was an improvement of 10 degrees of hip flexion range of motion with both the Unassisted and Assisted PNF stretching groups. There was no change in the control group from pre to post testing. These improvements mean that a 6-week, 12 session contract-relax (3 repetitions, 10-second passive stretch, 7-second isotonic contraction, 10-second passive stretch, 10-second rest) PNF stretching intervention significantly improved hip flexion range of motion. The stretching intervention did not induce changes to hamstring MVIC or vertical jump height in this study, which also supports our hypothesis that there would not be a decline in performance measures following the Unassisted and Assisted PNF stretching protocols.

The results of the present study are consistent with previous research regarding PNF stretching that has reported that it is effective in increasing range of motion across various joints, especially the hip joint. ${ }^{6-8,10,12-14,16,76}$ Previous research also states that PNF stretching is more effective than static stretching, ${ }^{4,8,14,16-18,76-78}$ which is why the current study investigated PNF stretching. A study by Rowlands ${ }^{16}$ found that over a 6 week time period, PNF stretching significantly increased joint range of motion by 32 degrees using a contract relax antagonist contract PNF stretching technique. By incorporating a quadriceps contraction in the PNF stretching protocol targeting the hamstring muscles, the idea of reciprocal inhibition which may 
be responsible for significant increases in range of motion of 32 degrees compared to the 10 degrees reported in the present study. For this study, we utilized the contract-relax technique, which has been effective in increasing hamstring flexibility. ${ }^{62,73}$ All participants included in this study possessed tight or restricted hamstrings, which was appropriate for the contract-relax technique as one of its indications is when range of motion is limited due to muscle tightness. ${ }^{63}$ Specifically, the contract-relax technique used in the current study has previously resulted in a significant 7-degree increase in hip flexion range of motion in a six week investigation. ${ }^{62}$ The results can provide rationale for using contract-relax PNF stretching to target hamstring flexibility and evidence to support its use.

The neurophysiological mechanisms responsible for the effect that stretching, specifically PNF stretching, has on muscle flexibility remains under some debate. PNF as a stretching and strengthening intervention increases the length and the neuromuscular efficiency of the muscle. ${ }^{12}$ Many other factors may influence the potential effectiveness of a PNF stretching protocol such as, the antagonist versus agonist muscle contraction, duration of contraction and relaxation after contraction, contraction intensity and duration, stretch duration, contraction intensity, and use of an experienced. ${ }^{58}$ The effectiveness of the contract-relax PNF stretching technique has been supported by the idea of facilitating autogenic inhibition or through increased stretch tolerance over time. Autogenic inhibition is described as a reflexive inhibition (relaxation) of the muscle that is experiencing a stretch or forceful contraction (tension) and is due to the inhibitory effect of the golgi tendon organ (GTO) in the tendon. ${ }^{8,19,59}$ The contract-relax PNF stretching technique utilized in this study incorporated a passive stretch and active isotonic muscle contraction of the hamstrings which increases the tension sensed by the GTO and a subsequent reflexive 
inhibition. ${ }^{60}$ Ultimately, this relaxation of the hamstrings can lead to decreased neural activity, which allows for increased stretch, flexibility, and range of motion. ${ }^{8,16}$

On the other hand, both the Unassisted and Assisted PNF stretch interventions required 12 sessions, which could lead to an increased stretch tolerance for the participants. ${ }^{10,24}$ Stretch tolerance can be explained by re-introducing the muscle spindle and relaxation of the muscle by inhibiting the muscle spindles of the muscle being stretched. The muscle spindles are the first to respond when a muscle is stretched. The muscle spindles send a sensory signal to the spinal cord, which reflexively responds with a muscle contraction of the same muscle being stretched. This is the stretch reflex. Over time, with more stretching, it takes a bit longer for the muscle spindle to be activated, therefore participants possess and increased stretch tolerance. This increased stretch tolerance can result in greater joint range of motion. Sharman ${ }^{8}$ stated that the exact mechanism behind the increase in ROM has not been identified but decreased pain levels is a possibility. ${ }^{79}$ This decreased pain could allow for further relaxation of the hamstring muscle group. The decreased discomfort allows for a decreased neural response to the stretch which does not stimulate pain responses and allows for an increased stretch tolerance. While autogenic inhibition or increased stretch tolerance is responsible for the improvements in range of motion in contract-relax PNF stretching remains unknown, the combination of neurophysiological mechanisms and potential decreased pain is beneficial for the patient as well as for support for PNF stretching techniques.

An additional objective of this study was to investigate the effect that the contract-relax PNF protocol had on athletic measures such as strength and vertical jump height. This study focused on hamstring strains and the factors that surround the prevalence of this injury in elite soccer players. A typical soccer match or training session involves many athletic movements 
from sprinting to kicking to jumping, which is why this study investigated how PNF stretching may affect some of these athletic maneuvers. Hamstring strength deficits have been identified as a risk factor for hamstring strains, which is an added reason for measuring hamstring strength. ${ }^{1,4,5,77}$ Furthermore, previous research has investigated strength using the MVIC following PNF stretch interventions and results remain inconclusive. Our results are consistent with a study that found minor, insignificant decreases in quadriceps strength in indoor soccer players following a PNF stretch intervention. ${ }^{26}$ The study by Reis differs from the present study in that the indoor soccer players in an acute PNF stretch intervention and focused on the quadriceps whereas this study was long-term over a 6-week duration and focused on the hamstrings. Furthermore, the present study completed the post-testing no more than 1-week following the last stretch intervention and did not measure MVIC immediately following the last stretch intervention. Conversely, Yutetsu et al. ${ }^{25}$ found that PNF stretching significantly impaired hamstring maximum voluntary contraction by $7.1 \%$. The present study suggests that a long-term PNF stretching intervention does not significantly impair hamstring strength and would not increase the risk for sustaining a hamstring strain.

Vertical jump height is another athletic measure that has been negatively impacted by PNF stretching in previous research. ${ }^{21}$ Some ideas include a decrease in musculotendinous stiffness or that the stretching causes muscular inhibition due to stretching the muscle too far outside of its capacity. ${ }^{12}$ Therefore, a 6-week stretching protocol did not impair or enhance vertical jump height. These results support previous research that has investigated vertical jump ability following PNF stretching and resulted in no significant findings. ${ }^{22-24,62}$ All four of these studies measured vertical jump height and ability immediately following the stretch interventions which differs from the present study that completed post-testing within 1-week of the last 
intervention. This study's results contradict a previous study by Church et al. ${ }^{21}$ that found that PNF stretching significantly impaired vertical jump height measures. Ultimately, this study found that contract-relax PNF stretching could significantly increase range of motion without consequently impairing hamstring strength and vertical jump ability. This applies well for elite soccer players in targeting a known risk factor for injury, flexibility, yet will not affect their ability to perform all sport related tasks during a match or practice.

The final objective of this study is the comparison of the Unassisted and Assisted PNF stretching protocols and whether both promote similar changes in range of motion and hamstring flexibility. Typically, PNF stretching is completed with the use of a partner yet that can become time consuming for clinicians who may need to stretch many patients. A few studies have introduced the use of a wall, chair, or a stretch strap as utilized in this study. ${ }^{9,10,17,19,24,58}$ This can be important for clinicians who may want to utilize PNF stretching but do not possess the luxury of time or have multiple other athletes to treat. Furthermore, this study is one of two that utilized a Theraband Dynamic Stretch Strap to aid in the completion of the Unassisted PNF stretching protocol. ${ }^{10}$ Schuback et al. ${ }^{17}$ compared an Unassisted PNF stretching protocol and an assisted group and found that both groups improved on hip flexion ROM measures and possessed the potential to be used interchangeably to target hamstring flexibility but did not utilize a stretch strap. Maddigan et al. ${ }^{10}$ brought on further investigation into the comparison of unassisted and assisted techniques by introducing the stretch strap as a tool for completing unassisted or selfPNF. She found that unassisted and assisted PNF stretching groups possessed similar improvements in hip flexion range of motion. We found similar improvements in hip flexion ROM with both PNF stretch groups. Unlike the Maddigan ${ }^{10}$ study, which found no significant improvements in the Unassisted stretching, our study found not only improvements in ROM but 
also ROM improvements very comparable to the Assisted PNF group. The novelty of the current study is that we investigated range of motion and athletic measures consistent with the intended population of elite soccer players rather than reaction and movement time. This provides further support for the idea that the contract-relax Unassisted PNF stretching protocol used in the present study can be used in place of a partner-assisted stretching program without compromising flexibility improvements.

This study demonstrated that contract-relax PNF stretching is appropriate for improving flexibility while not greatly impairing athletic performance measures of hamstring strength and vertical jump performance. Furthermore, clinicians can properly instruct their athletes and patients on how to complete the contract-relax PNF stretching protocol used in this study and trust that the athletes will benefit from the Unassisted stretching just as well as if the clinician stretched them. This gives clinicians the ability to focus on treating their other patients without the time-consuming task of stretching multiple athletes.

This study has some limitations despite its significant results including the use of a convenient sample from local universities. It is hard to generalize our results and conclusions to the specific population of elite soccer players when a variety of healthy, physically active participants were utilized in this study. With that said, it is hard to control what sort of training participants completed outside of the stretching sessions which was not controlled. This was decided on as most flexibility training programs are designed to be reinforced with strength, agility, and sport specific training programs. Another limitation is the time duration between the last stretch session and the post-test session. Initially, the idea was to complete the post-test sessions 24 to 48 hours after the last stretch session. Yet, due to the design of the study and 
attempting to observe longer lasting flexibility gains, this study benefitted from the longer period between post-testing in that both PNF groups significantly benefitted even after a week.

Future research could investigate the effects of Unassisted and Assisted PNF stretching on a soccer specific population, which would make generalizing results easier to the elite soccer population. With that, soccer specific performance measures could be measured including kicking speed and an agility measure such as a T-test or shuttle run. Researchers could continue with another 6-week intervention yet complete post measures of athletic performance measures immediately following the first stretching session, at the 3-week mark, and final post-test measures at the conclusion of the 6 weeks. 


\section{REFERENCES}

1. Beijsterveldt AMC, Port IGL, Vereijken AJ, Backx FJG. Risk Factors For Hamstring Injuries In Male Soccer Players: A Systematic Review Of Prospective Studies.

Scandinavian Journal Of Medicine \& Science In Sports. 2013;23(3):253-262.

2. Jing-Chao Han, Hui Liu. Meta-Analysis Of Risk Factors For Hamstring Muscle Injury In Athletes. Journal Of Beijing Sport University. 2015;38(2):79-86.

3. Beijsterveldt AMC, Port IGL, Vereijken AJ, Backx FJG. Risk Factors For Hamstring Injuries In Male Soccer Players: A Systematic Review Of Prospective Studies. Scandinavian Journal Of Medicine \&Amp; Science In Sports. 2013;23(3):253-262.

4. Freckleton G, Pizzari T. Risk Factors For Hamstring Muscle Strain Injury In Sport: A Systematic Review And Meta-Analysis. British Journal Of Sports Medicine. 2013;47(6):1-9.

5. Turner An, Cree J, Comfort P, Et Al. Hamstring Strain Prevention In Elite Soccer Players. Strength \& Conditioning Journal (Lippincott Williams \& Wilkins). 2014;36(5):10-20.

6. Ahmed H, Iqbal A, Anwer S, Alghadir A. Effect Of Modified Hold-Relax Stretching And Static Stretching On Hamstring Muscle Flexibility. Journal Of Physical Therapy Science. $2015 ; 27(2): 535-538$.

7. Kyoung-Il Lim, Hyung-Chun Nam, Kyoung-Sim J. Effects On Hamstring Muscle Extensibility, Muscle Activity, And Balance Of Different Stretching Techniques. Journal Of Physical Therapy Science. 2014;26(2):209-213.

8. Sharman MJ, Cresswell AG. Proprioceptive Neuromuscular Facilitation Stretching: Mechanisms And Clinical Implications. Sports Medicine. 2006;36(11):929-939. 
9. Wicke J, Gainey K, Figueroa M. A Comparison Of Self-Administered Proprioceptive Neuromuscular Facilitation To Static Stretching On Range Of Motion And Flexibility. Journal Of Strength \& Conditioning Research (Lippincott Williams \& Wilkins). 2014;28(1):168-172.

10. Maddigan ME, Peach AA, Behm DG. A Comparison Of Assisted And Unassisted Proprioceptive Neuromuscular Facilitation Techniques And Static Stretching. Journal Of Strength \& Conditioning Research (Lippincott Williams \& Wilkins). 2012;26(5):12381244.

11. Konrad A, Gad M, Tilp M. Effect Of Pnf Stretching Training On The Properties Of Human Muscle And Tendon Structures. Scandinavian Journal Of Medicine \& Science In Sports. 2015;25(3):346-355.

12. Hindle KB, Whitcomb TJ, Briggs WO, Junggi H. Proprioceptive Neuromuscular Facilitation (Pnf): Its Mechanisms And Effects On Range Of Motion And Muscular Function. Journal Of Human Kinetics. 2012;31:105-113.

13. Wallin D, Ekblom B, Grahn R, Nordenborg T. Improvement Of Muscle Flexibility. A Comparison Between Two Techniques. The American Journal Of Sports Medicine. 1985;13(4):263-268.

14. Lucas RC, Koslow R. Comparative Study Of Static, Dynamic, And Proprioceptive Neuromuscular Facilitation Stretching Techniques On Flexibility. Perceptual \& Motor Skills. 1984;58(2):615-618.

15. Funk DC, Swank AM, Mikla BM, Fagan TA, Farr BK. Impact Of Prior Exercise On Hamstring Flexibility: A Comparison Of Proprioceptive Neuromuscular Facilitation And 
Static Stretching. Journal Of Strength \& Conditioning Research (Allen Press Publishing Services Inc). 2003;17(3):489-492 484p.

16. Rowlands AV, Marginson VF, Lee J. Chronic Flexibility Gains: Effect Of Isometric Contraction Duration During Proprioceptive Neuromuscular Facilitation Stretching Techniques. Research Quarterly For Exercise \& Sport. 2003;74(1):47-51.

17. Schuback B, Hooper J, Salisbury L. A Comparison Of A Self-Stretch Incorporating Proprioceptive Neuromuscular Facilitation Components And A Therapist-Applied PnfTechnique On Hamstring Flexibility. Physiotherapy. 2004;90(3):151-157.

18. Houglum PA. Therapeutic Exercise For Musculoskeletal Injuries. Champaign, Il : Human Kinetics, C2010. 3rd Ed.; 2010.

19. Prentice WE, Kaminski TW. Rehabilitation Techniques For Sports Medicine And Athletic Training. Mcgraw-Hill New York:; 2004.

20. Feland JB, Marin HN. Effect Of Submaximal Contraction Intensity In Contract-Relax Proprioceptive Neuromuscular Facilitation Stretching. Br J Sports Med. 2004;38(4):E18.

21. Church JB, Wiggins MS, Moode FM, Crist R. Effect Of Warm-Up And Flexibility Treatments On Vertical Jump Performance. Journal Of Strength \& Conditioning Research (Allen Press Publishing Services Inc). 2001;15(3):332-336 335p.

22. Young W, Elliott S. Acute Effects Of Static Stretching, Proprioceptive Neuromuscular Facilitation Stretching, And Maximum Voluntary Contractions On Explosive Force Production And Jumping Performance. Research Quarterly For Exercise And Sport. 2001;72(3):273-279.

23. Christensen BK, Nordstrom BJ. The Effects Of Proprioceptive Neuromuscular Facilitation And Dynamic Stretching Techniques On Vertical Jump Performance. Journal 
Of Strength \& Conditioning Research (Lippincott Williams \& Wilkins). 2008;22(6):18261831.

24. Place N, Blum Y, Armand S, Maffiuletti NA, Behm DG. Effects Of A Short Proprioceptwe Neuromuscular Facilitation Stretching Bout On Quadriceps Neuromuscular Function, Flexibility, And Vertical Jump Performance. Journal Of Strength \& Conditioning Research (Lippincott Williams \& Wilkins). 2013;27(2):463-470.

25. Yutetsu M, Hisashi N, Yuji O, Shizuo K, Junichiro A. Effects Of Proprioceptive Neuromuscular Facilitation Stretching And Static Stretching On Maximal Voluntary Contraction. Journal Of Strength \& Conditioning Research (Lippincott Williams \& Wilkins). 2013;27(1):195-201 197p.

26. Reis Edfs, Pereira GB, Sousa NmF, et al. Acute Effects Of Proprioceptive Neuromuscular Facilitation And Static Stretching On Maximal Voluntary Contraction And Muscle Electromyographical Activity In Indoor Soccer Players. Clinical Physiology \& Functional Imaging. 2013;33(6):418-422.

27. Starkey C, Brown SD, Ryan JL. Orthopedic And Athletic Injury Examination Handbook. [Electronic Resource]. Philadelphia : F.A. Davis Co., C2010. Ed. 2.; 2010.

28. Schache AG, Crossley KM, Macindoe IG, Fahmer BB, Pandy MG. Can A Clinical Test Of Hamstring Strength Identify Football Players At Risk Of Hamstring Strain? Knee Surgery, Sports Traumatology, Arthroscopy. 2011;19(1):38-41.

29. Made A, Wieldraaijer T, Kerkhoffs G, et al. The Hamstring Muscle Complex. Knee Surgery, Sports Traumatology, Arthroscopy. 2015;23(7):2115-2122. 
30. Ekstrand J, Lee JC, Healy JC. Mri Findings And Return To Play In Football: A Prospective Analysis Of 255 Hamstring Injuries In The Uefa Elite Club Injury Study. British Journal Of Sports Medicine. 2016;50(12):738-744 737p.

31. Askling CM, Malliaropoulos N, Karlsson J. High-Speed Running Type Or StretchingType Of Hamstring Injuries Makes A Difference To Treatment And Prognosis. British Journal Of Sports Medicine. 2012;46(2):86-87.

32. Askling CM, Tengvar M, Saartok T, Thorstensson A. Acute First-Time Hamstring Strains During Slow-Speed Stretching Clinical, Magnetic Resonance Imaging, And Recovery Characteristics. American Journal Of Sports Medicine. 2007;35(10):17161724.

33. Brukner P. Hamstring Injuries: Prevention And Treatment-An Update. British Journal Of Sports Medicine. 2015;49(19):1241-1244.

34. Askling C, Saartok T, Thorstensson A. Type Of Acute Hamstring Strain Affects Flexibility, Strength, And Time To Return To Pre-Injury Level. British Journal Of Sports Medicine. 2006;40(1):40-44.

35. Askling CM, Tengvar M, Saartok T, Thorstensson A. Acute First-Time Hamstring Strains During High-Speed Running A Longitudinal Study Including Clinical And Magnetic Resonance Imaging Findings. American Journal Of Sports Medicine. 2007;35(2):197-206.

36. Sherry MA, Johnston TS, Heiderscheit BC. Rehabilitation Of Acute Hamstring Strain Injuries. Clinics In Sports Medicine. 2015;34(2):263-284. 
37. Thelen DG, Chumanov ES, Hoerth DM, et al. Hamstring Muscle Kinematics During Treadmill Sprinting. Medicine \&Amp; Science In Sports \&Amp; Exercise. 2005;37(1):108-114.

38. Goldman EF, Jones DE. Interventions For Preventing Hamstring Injuries: A Systematic Review. Physiotherapy. 2011;97(2):91-99.

39. Hawkins RD, Fuller CW. A Prospective Epidemiological Study Of Injuries In Four English Professional Football Clubs...Including Commentary By Mccann G. British Journal Of Sports Medicine. 1999;33(3):196-203 198p.

40. Arnason A, Andersen TE, Holme I, Engebretsen L, Bahr R. Prevention Of Hamstring Strains In Elite Soccer: An Intervention Study. Scandinavian Journal Of Medicine \& Science In Sports. 2008;18(1):40-48.

41. Koulouris G, Connell Da, Brukner P, Schneider-Kolsky M. Magnetic Resonance Imaging Parameters For Assessing Risk Of Recurrent Hamstring Injuries In Elite Athletes. American Journal Of Sports Medicine. 2007;35(9):1500-1506.

42. Shrier I. Does Stretching Improve Performance? A Systematic And Critical Review Of The Literature. Clinical Journal Of Sport Medicine. 2004;14(5):267-273.

43. Ingraham SJ. The Role Of Flexibility In Injury Prevention And Athletic Performance: Have We Stretched The Truth? Minnesota Medicine. 2003;86(5):58-61.

44. Orchard J, Best Tm. The Management Of Muscle Strain Injuries: An Early Return Versus The Risk Of Recurrence. (Guest Editorial). Clinical Journal Of Sport Medicine. 2002;12(1):3-5. 
45. Petersen J, Thorborg K, Nielsen MB, Hölmich P. Acute Hamstring Injuries In Danish Elite Football: A 12-Month Prospective Registration Study Among 374 Players. Scandinavian Journal Of Medicine \& Science In Sports. 2010;20(4):588.

46. Petersen J, Thorborg K, Nielsen MB, Budtz-Jørgensen E, Hölmich P. Preventive Effect Of Eccentric Training On Acute Hamstring Injuries In Men's Soccer: A ClusterRandomized Controlled Trial. American Journal Of Sports Medicine. 2011;39(11):22962303.

47. Prentice We. Principles Of Athletic Training : A Competency-Based Approach. McgrawHill; 2009.

48. Hägglund MA, Waldén MA, Ekstrand JA, Linköpings Universitet Ifmohsofo, Linköpings Universitet Ho. Injuries Among Male And Female Elite Football Players. Scandinavian Journal Of Medicine And Science In Sports. 2009:819.

49. Alway SE. Force And Contractile Characteristics After Stretch Overload In Quail Anterior Latissimus Dorsi Muscle. Journal Of Applied Physiology. 1994;77(1):135-141.

50. Mann D, Whedon C. Functional Stretching: Implementing A Dynamic Stretching Program. Athletic Therapy Today. 2001;6(3):10-13.

51. Puentedura EJ, Huijbregts PA, Celeste S, et al. Immediate Effects Of Quantified Hamstring Stretching: Hold-Relax Proprioceptive Neuromuscular Facilitation Versus Static Stretching. Physical Therapy In Sport. 2011;12(3):122-126.

52. Alter MJ. Science Of Flexibility. Champaign, Il : Human Kinetics, C1996. 2nd Ed.; 1996.

53. Etnyre BR, Abraham LD. Gains In Range Of Ankle Dorsiflexion Using Three Popular Stretching Techniques. American Journal Of Physical Medicine. 1986;65(4):189-196. 
54. Ferber R, Osternig L, Gravelle D. Effect Of Pnf Stretch Techniques On Knee Flexor Muscle Emg Activity In Older Adults. Journal Of Electromyography \& Kinesiology. 2002;12(5):391-397.

55. Magnusson SP, Simonsen EB, Aagaard P, Dyhre-Poulsen P, Mchugh MP, Kjaer M. Mechanical And Physiological Responses To Stretching With And Without Preisometric Contraction In Human Skeletal Muscle. Archives Of Physical Medicine \& Rehabilitation. 1996;77(4):373-378 376p.

56. Moore MA. An Electromyographic Investigation Of Muscle Stretching Techniques [Microform] / By Marjorie Ann Moore. 1979.; 1982.

57. Sady SP, Wortman M, Blanke D. Flexibility Training: Ballistic, Static Or Proprioceptive Neuromuscular Facilitation? Archives Of Physical Medicine \& Rehabilitation. 1982;63(6):261-263.

58. Sheard PW, Paine TJ. Optimal Contraction Intensity During Proprioceptive Neuromuscular Facilitation For Maximal Increase Of Range Of Motion. Journal Of Strength \& Conditioning Research (Lippincott Williams \& Wilkins). 2010;24(2):416-421.

59. Laporte Y, Lloyd DPC. Nature And Significance Of The Reflex Connections Established By Large Afferent Fibers Of Muscular Origin. The American Journal Of Physiology. 1952;169(3):609-621.

60. Etnyre BR, Lee EJ. Comments On Proprioceptive Neuromuscular Facilitation Stretching Techniques. Research Quarterly For Exercise \& Sport. 1987;58(2):184-188.

61. Etnyre BR, Lee EJ. Chronic And Acute Flexibility Of Men And Women Using Three Different Stretching Techniques. / La Souplesse Chronique Et Aigue Chez Des Hommes 
Et Des Femmes Utilisant Trois Techniques D ' Etirement Differentes. Research Quarterly For Exercise \&Amp; Sport. 1988;59(3):222-228.

62. Yuktasir B, Kaya F. Investigation Into The Long-Term Effects Of Static And Pnf Stretching Exercises On Range Of Motion And Jump Performance. Journal Of Bodywork \& Movement Therapies. 2009;13(1):11-21.

63. Akbulut T, Agopyan A. Effects Of An Eight-Week Proprioceptive Neuromuscular Facilitation Stretching Program On Kicking Speed And Range Of Motion In Young Male Soccer Players. Journal Of Strength And Conditioning Research / National Strength \& Conditioning Association. 2015;29(12):3412-3423.

64. Dong Ho K, Young Uk R. Applying Proprioceptive Neuromuscular Facilitation Stretching: Optimal Contraction Intensity To Attain The Maximum Increase In Range Of Motion In Young Males. Journal Of Physical Therapy Science. 2015;27(7):2129-2132.

65. Escolar DM, Henricson EK, Mayhew J, et al. Clinical Evaluator Reliability For Quantitative And Manual Muscle Testing Measures Of Strength In Children. Muscle \&Amp; Nerve. 2001;24(6):787-793.

66. Cengiz A. EMG And Peak Force Responses To Pnf Stretching And The Relationship Between Stretching-Induced Force Deficits And Bilateral Deficits. Journal Of Physical Therapy Science. 2015;27(3):631-634.

67. Osternig LR, Robertson R, Troxel R, Hansen P. Muscle Activation During Proprioceptive Neuromuscular Facilitation (Pnf) Stretching Techniques... Stretch-Relax (Sr), Contract-Relax (Cr) And Agonist Contract-Relax (Acr). American Journal Of Physical Medicine. 1987;66(5):298-307 210p. 
68. Laudner KG, Benjamin PJ, Selkow NM. Effect Of Ankle Positioning During Hamstring Stretches For Improving Straight Leg Hip Flexion Motion. Clinical Journal Of Sport Medicine. 2016;26(2):167-171.

69. Worrell TW, Kart G, Adamczyk D, Moore R. Influence Of Joint Position On Electromyographic And Torque Generation During Maximal Voluntary Isometric Contractions Of The Hamstrings And Gluteus Maximus Muscles. Journal Of Orthopaedic \&Amp; Sports Physical Therapy. 2001;31(12):730-740.

70. Nedelec M, Mccall A, Carling C, Legall F, Berthoin S, Dupont G. The Influence Of Soccer Playing Actions On The Recovery Kinetics After A Soccer Match. Journal Of Strength \& Conditioning Research (Lippincott Williams \& Wilkins). 2014;28(6):15171523.

71. Acero RM, Sánchez JA, Fernández-Del-Olmo M. Tests Of Vertical Jump: Countermovement Jump With Arm Swing And Reaction Jump With Arm Swing. Strength \& Conditioning Journal (Lippincott Williams \& Wilkins). 2012;34(6):87-93.

72. Etnyre BR, Lee EJ. Chronic And Acute Flexibility Of Men And Women Using Three Different Stretching Techniques. / La Souplesse Chronique Et Aigue Chez Des Hommes Et Des Femmes Utilisant Trois Techniques D ' Etirement Differentes. Research Quarterly For Exercise \& Sport. 1988;59(3):222-228.

73. Surburg PR, Schrader JW. Proprioceptive Neuromuscular Facilitation Techniques In Sports Medicine: A Reassessment. Journal Of Athletic Training (National Athletic Trainers' Association). 1997;32(1):34.

74. Jaric S. Muscle Strength Testing: Use Of Normalisation For Body Size. Sports Med. 2002;32(10):615-631. 
75. Folland JP, Mc Cauley TM, Williams AG. Allometric Scaling Of Strength Measurements To Body Size. Eur J Appl Physiol. 2008;102(6):739-745.

76. Handel M, Horstmann T, Dickhuth HH, Guelch RW. Effects Of Contract-Relax Stretching Training On Muscle Performance In Athletes. / Effets D ' Une Seance D ' Etirements Contractes Et Relaches Sur La Performance Musculaire De L ' Athlete. European Journal Of Applied Physiology \& Occupational Physiology. 1997;76(5):400408.

77. Navarro E, Chorro D, Torres G, García C, Navandar A, Veiga S. A Review Of Risk Factors For Hamstring Injury In Soccer: A Biomechanical Approach. Motricidad: European Journal Of Human Movement. 2015;34:52.

78. Gribble PA, Guskiewitz KM, Prentice WA, Shields EW. Effects Of Static And HoldRelax Stretching On Hamstring Range Of Motion Using The Flexability Le1000. Journal Of Sport Rehabilitation. 1999;8(3):195.

79. Magnusson SP, Simonsen EB, Aagaard P, Boesen J, Johannsen F, Kjaer M. Determinants Of Musculoskeletal Flexibility: Viscoelastic Properties, Cross-Sectional Area, Emg And Stretch Tolerance. Scand J Med Sci Sports. 1997;7(4):195-202. 


\title{
APPENDIX A: IRB INFORMED CONSENT
}

\section{ILLINOIS STATE UNIVERSITY INFORMED CONSENT}

\author{
Comparison of Self-Directed PNF Stretching versus Clinician-Assisted PNF Stretching on Hip \\ Range of Motion, Maximum Voluntary Isometric Contraction, \& Maximum Vertical Jump Height.
}

Principal Investigator:

Rebecca Begalle, PhD, ATC

Illinois State University

School of Kinesiology \& Recreation

Campus Box 5120

Normal, IL 61790

309-438-2605
Joel T Lockhart, ATC

Skip Williams, Ph.D

Noelle Selkow, Ph.D

Matt Kovarik

Bridget Hill

\section{STUDY DESCRIPTION}

The purpose of this study is to investigate the effectiveness of two forms of stretching techniques using proprioceptive neuromuscular facilitation (PNF). We aim to study whether PNF stretching performed by yourself (Unassisted) or PNF stretching assisted by someone else (Assisted) is more effective when considering hamstring muscle flexibility, hamstring muscle contraction, and vertical jump height. We hope the results will help determine the most effective way to using this stretch.

\section{SUBJECTS}

You are being asked to participate in this research project because you are a recreationally active college student between the ages of 18 and 30 years old. While there is no direct benefit to you for participating in this study, you may obtain improvements in hamstring flexibility from one of the stretching interventions.

\section{PROCEDURES}

Each participant will attend the first session to determine if they have tightness in their hamstring muscle that would qualify them for this study. If so, they will continue with the remainder of the pretesting protocol and group assignment for the stretching intervention.

Each testing session (pre- and post-testing) will last approximately 30 minutes. Each participant will also attend 11 additional training sessions to perform a 15-minute stretching protocol. In total, participation in this study will require approximately 4.5 hours of time over a period of 6 weeks if placed in a treatment group. If placed in the control group, a total of 1 hour of time will be required. All testing will occur either in the SMART clinic (ISU) or Shirk Center (IWU). Each participant will provide the investigators with their age, height, body mass, and history of lower extremity injury. Additional demographics, such as dominant kicking leg and leg segment length will also be recorded. All participants will wear comfortable athletic clothing and athletic shoes to the testing site(s).

Screening: To assess hamstring flexibility, and potential eligibility, each participant will be positioned on their back on a treatment table with both legs fully relaxed on the table. Prior to the flexibility assessment, all participants will perform a 5-minute warm-up on a stationary bike at 50 rotations per minute (RPM). The investigator will move the test leg into straight leg hip flexion (towards the ceiling) until resistance is felt. Once this end point is determined, the investigator will measure the hip flexion angle using a digital inclinometer (small plastic device) aligned with the hip and leg. Participants with a hip flexion angle of $\leq 90^{\circ}$ on both legs will be eligible to participate in this study. Any participant with a hip flexion angle that exceeds $90^{\circ}$ will be thanks for their time and excused from further participation. 
Pre-Test: To measure leg length each participant will be positioned on their back on a treatment table. The distance will be measured from the knee joint to the outside (lateral) ankle bone. This measurement will be completed and recorded for both legs using a cloth tape measure.

Then two additional measurements will be performed during the pre-test session; including muscle strength (maximum voluntary isometric contraction (MVIC)) and maximum vertical jump height.

To assess hamstring flexibility, the same measurements recorded during the screening process will be utilized as the pre-test measure. The three measurements will be carried over and recorded for each leg. There will be a 30 -second rest period in between each trial.

To assess hamstring muscle activation (MVIC) measurements, participants will be positioned on their stomach (prone) on the treatment table. The knee of the test leg will be bent to a 90 degree angle. A tester will stand behind the table on the same side as the test leg. The handheld dynamometer (small plastic device) will be positioned on the back of the lower leg just above the ankle to provide resistance and measure the muscle contraction. Each participant will be instructed to pull their foot towards their butt as hard as they can, pulling against the resistance provided by the tester for five seconds. Strong verbal encouragement will be provided throughout the 5 -second muscle contraction to encourage the most forceful contraction. This procedure will be completed three times on each leg with a full 60 -second rest period in between each trial.

To assess maximum vertical jump height, participants will be instructed to stand underneath a pole that has horizontal plastic pegs that stick out to measure jump height (Vertec system). Participants will stand with one arm raised overhead as high as possible. The tester will adjust the Vertec so that the lowest plastic peg touches the tallest point on the participants' hand. The participant will then be instructed to perform a counter movement jump and allowed one practice attempt to familiarize themselves with the task. The counter movement jump is performed by starting standing upright with their feet shoulders width apart and arms relaxed by their sides. When instructed to, the participants will complete a rapid countermovement by squatting down to a chair-sitting position. Once in a squat position, the vertical jump begins with an explosive extension of the legs in an attempt to jump and achieve the highest vertical height possible. The arms will swing up in an attempt to reach up as high as possible while completing the jump. The participant will reach and extend their arms as far as possible trying to reach the maximum height on the Vertec Jump Height Measuring System. Three jumps will be performed with a 30 -second rest in between each.

Upon completion of pre-testing, participants will be randomly assigned to one of three groups; Unassisted stretching, Assisted stretching, or the control group. If assigned to one of the stretching groups, participants will be instructed on their first stretching intervention immediately. If assigned to the control group, participants will be scheduled to return for post-test measurements 6-weeks later.

Intervention: The Unassisted PNF stretching group will use a canvas strap to help perform their stretches while receiving instructions from flyer on proper technique. The stretching routine will be performed by pulling the hip into flexion using the strap while keeping the knee straight. This stretch is held for 10 seconds followed by a 7-second muscle contraction of the hamstring moving the leg back down towards the table. Each participant will pull on the strap using their arms to provide resistance against the hamstring throughout the movement. The participant then relaxes and performs another 10second passive stretch using the stretch strap followed by a 10 second rest period. This process is completed 3 times per leg.

The Assisted PNF stretching group will perform the same stretching protocol, but will have a tester provide all passive stretching and resistance. The tester will passively move the hip into flexion (foot toward ceiling) until the point of first resistance. The stretch will be held for 10 seconds followed by a 7-second muscle contraction of the hamstring moving the leg back down towards the table. This is followed by another 10 -second passive hamstring stretch to a point of mild resistance. Afterwards, there will be a 10 second rest period. The routine is completed 3 times per leg per treatment intervention.

Post-test: Post-test measurements of hamstring flexibility, hamstring muscle contraction, and maximum vertical jump height will be completed following the final stretch intervention (session 13) or in week 6 for control participants. All testing procedures are the same as during pre-test. 


\section{RISKS}

As with any investigational study, there may be adverse events or side effects that are currently unknown and it is possible that certain of these unknown risks could be harmful. Previous human research studies have shown that the nature and number of adverse events associated with similar studies are rare. You may feel mild stretching during testing. If at any time you experience pain beyond the discomfort previously mentioned, you should inform the investigators and they will discontinue testing. In the rare instance that an injury does occur, the investigators involved with this study are trained to provide acute care for athletic related pain in the upper extremity. There is also a risk of loss of confidentiality; however, all data collected will be indicated by a participant number rather than their name to minimize this risk.

\section{CONFIDENTIALITY}

Any information about you obtained from this research will be kept as confidential (private) as possible. All records related to your involvement in this research study will be stored in a locked file cabinet. Your identity on these records will be indicated by a case number rather than by your name, and the information linking these numbers with your identity will be kept separate from the research records. Your research records will be destroyed when such is approved by primary investigator or at 5 years following study completion, whichever should occur first. You will not be identified by name in any publication of research.

Your participation in this study is voluntary and refusal to participate or discontinuing participation at any time will result in no penalty or loss of benefits.

\section{VOLUNTARY CONSENT:}

Any questions about this research project can be answered by the investigators listed on this form and any questions about your rights as a research participant can be answered by the Illinois State University Research Ethics \& Compliance office (309-438-2529). By signing this form, you agree to participate in this research study. A copy of this consent form will be given to you if requested. 


\section{APPENDIX B: DATA COLLECTION SHEET \\ Data Collection Sheet}

Subject \#:

Treatment Group:

Date:

Gender: (1) M (2) F Age:

Height (cm):

Weight $(\mathrm{kg})$ :

\section{Exclusion Criteria Questions:}

Are you physically active, performing exercise at least 3-5 days per week for a total of 90 minutes per week?

$\mathrm{Y} \quad \mathrm{N}$

Have you had a hamstring injury in the past 6 months?

$\mathrm{Y} \quad \mathrm{N}$

Have you had any lower extremity injury that has kept you out of participation for more than 2 days?

$\mathrm{Y} \quad \mathrm{N}$

Do have a history of lower extremity surgery?

Y N

Do you have any known neurological disorders that would make completing the required tasks difficult?

$\mathrm{Y} \quad \mathrm{N}$

Dominant Kicking leg?

R L

Screening: Left hip flexion ROM

Left leg length $(\mathrm{cm})$
Right hip flexion ROM

Right leg length $(\mathrm{cm})$

Baseline: Left hip flexion ROM

Right hip flexion ROM

Left leg MVIC (N) 1

2. 3.__ Right leg MVIC (N) 1. 2. 3. Vertical Jump Height (in) 1. 2. 3.

\section{Post-test: Left hip flexion ROM}

Right hip flexion ROM

Left leg MVIC (N) 2. 3. Right leg MVIC (N) 1. 2. 3. Vertical Jump Height (in) 1. 2. 3. 


\section{Unassisted PNF Stretch Protocol}

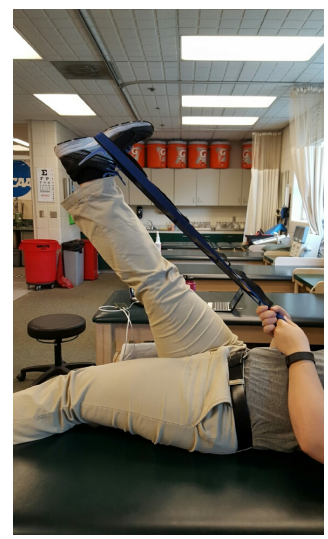

\section{Stretch \\ 10 \\ Seconds}

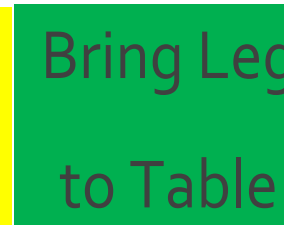

to Table

7 Seconds

S

1. 5-minute warm-up on a stationary bike

2. Place your foot into one of the loops of the Dynamic Stretch Strap.

3. Keeping your knee straight, use your arms to pull your hip and leg up in the air until you reach a point of resistance in your hamstrings.

4. Hold for 10 seconds using MetroTimer app to keep time

5. Following the 10 -second stretch, you will contract your hamstrings and try to bring your leg back down to the table. You will hold this contraction for a total of 7-seconds. Resist using arms and strap but allow leg to lower to table

6. Following the 7-second contraction, you are going to relax the leg being stretched and passively bring your leg into hip flexion using your arms and hold another 10-second stretch.

7. Following the second 10 -second stretch you will allow your leg to rest on the table for 10 seconds

8. Repeat $3 x$ on each leg 

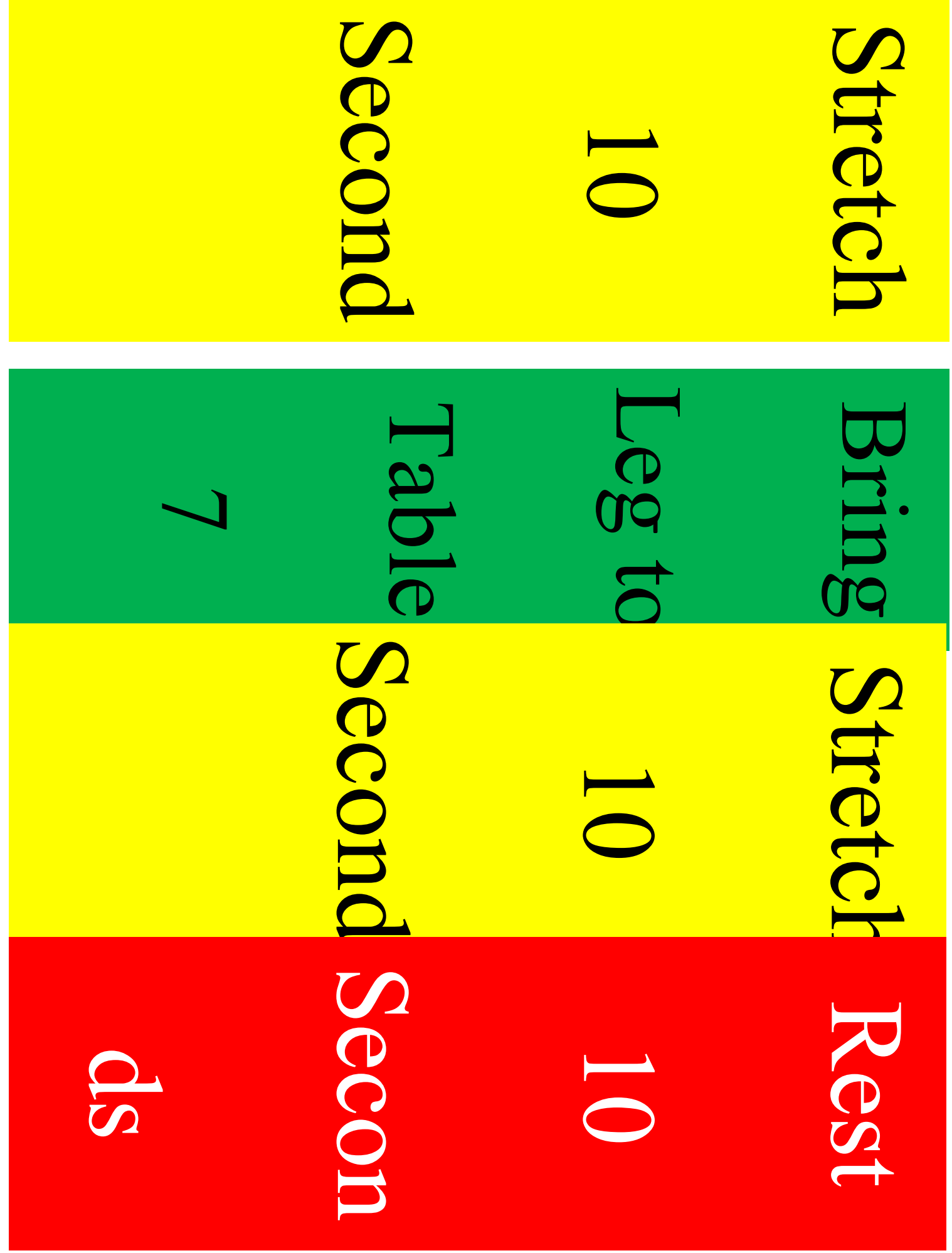